\title{
Etica, estetica e politica del cibo in Petrarca
}

\section{Enrico Fenzi}

enrico.fenzi@aleph.it

\begin{abstract}
Il saggio considera l'insieme degli atteggiamenti di Petrarca nei confronti del cibo, testimoniati da una lunga serie di passi sparsi nella sua opera, e in particolare in alcune importanti lettere «Senili». Da questo esame risultano varie funzioni assegnate al discorso sull'alimentazione: quella oppositiva, che distingue tra gozzoviglia e moderazione, tra cibi artefatti e cibi naturali, tra sporcizia e pulizia, e infine, sul piano sociale e politico, tra lusso e corruzione da una parte, e modestia e virtù dall'altro; quella positiva, che associa la buona e semplice alimentazione naturale alla salute e alla vita tranquilla e solitaria; quella estetica, non meno importante delle altre, che fa del modo di nutrirsi un "test" particolarmente importante sulla civiltà e la buona educazione. Cibarsi, insomma, è per Petrarca un fatto assolutamente culturale, e con ciò egli chiaramente supera i dilemmi della morale cristiana, e pone per primo le basi per una riflessione moderna sull'argomento.
\end{abstract}

Parole chiave: cibo, frutta fresca, acqua, pulizia, digiuno, salute, moderazione, gozzoviglia, obesità, malattia, corruzione.

\section{Abstract}

The research considers the combined attitudes of Petrarch in comparison with food, attested by a long series of scattered steps in his work and, in particular, in some significant «Senile» letters. There are various functions resulting from this research, aimed at the discussion of nutrition: The opposing, which distinguishes between gluttony and moderate eating, artificial and natural foods, cleanliness and dirtiness — on both a social and political level, between luxury and depravation, on the one hand, and modesty and virtue, on the other. The positive, associates a good, simple natural diet with health and a peaceful, private life. The aesthetic, of no less importance than the others, tests eating habits, proven particularly important with regard to civilisation and good education. In short, for Petrarch, eating was a completely cultural event, with which one clearly overcame Christian moral dilemmas and which firmly stood by its foundations for a modern reflection on the debate.

Key words: food, fresh fruit, water, fasting, health, moderation, gluttony, obesity, illness, depravation. 
«Non ho mangiato ma divorato un ottimo melone, senza farne parte con nessuno"; ${ }^{1}$ "Uva, fichi, noci, mandorle sono per me vere delizie, e mi piacciono i pesciolini dei quali questo fiume è ricco»; ${ }^{2}$ "vieni, e ti offrirò i succosi grappoli di questi colli pieni di viti, e fichi dolcissimi e acqua fresca appena attinta dal cuore della sorgente»... ${ }^{3}$

Sono appena tre citazioni tra le molte possibili (e su di esse dovrò tornare), ma già bastano ad attirare la nostra attenzione su un fatto che non so se sia stato sin qui osservato con la cura che merita. Petrarca parla molte volte, anche se spesso in maniera rapida, del cibo, e quasi sempre fa delle sue abitudini alimentari un elemento importante del ritratto di sé che attraverso le sue opere egli ha pazientemente cercato di delineare. Andiamo alle prime pagine $(\$ 5)$ di un testo per dire così istituzionale com’è la Posteritati, e leggiamo, in un contesto appena più ampio:

Sono stato un grande sprezzatore delle ricchezze, non perché non le desiderassi ma perché odiavo le fatiche e le preoccupazioni che ne sono le compagne inseparabili: e avrebbero fatto sì che mi toccasse anche il fastidio aggiuntivo di dover offrire lauti banchetti. Ma in verità, mangiando poco e in maniera semplice, ho vissuto meglio di quanto abbiano fatto tutti gli imitatori di Apicio con i loro squisitissimi piatti. Non mi sono mai piaciuti quelli che vengono definiti «conviti» e sono invece delle scorpacciate nemiche della moderazione e della buona educazione. Ho sempre pensato che fosse noioso e inutile invitare qualcuno solo per questo, così come l'essere invitato: ed è invece così bello stare in intimità con gli amici che il loro arrivo è sempre stata la mia più grande gioia, e mai per mia scelta ho mangiato da solo. ${ }^{4}$

1. Disp. 2: «Peponem optimum non comedi sed devoravi, nemine in partem admisso». Da F. Petrarca, Lettere disperse. A cura di Alessandro Pancheri, Parma: Fond. Pietro Bembo/Guanda Editore, 1994, p. 8 (=Var. 30).

2. Fam. XIII 8, 9: «Uva ficus nuces amygdale delitie mee sunt; quibus hic fluvius abundat, pisciculis delector».

3. Epyst. III 1, 108-110, a Giovanni Colonna: «nos tibi pampineos colles gravidosque racemos / denique mellifluas ficus undamque recentem / gurgite de medio offerimus». Dal testo a cura di Enrico Bianchi, nel volume Rime, Trionfi e poesie latine..., Milano-Napoli: Ricciardi, 1951 (avanti, le citazioni dalle Epystole senza altra indicazione s'intendono derivate di qui).

4. «Divitiarum contemptor eximius, non quod divitias non optarem, sed labores curasque oderam, opum inseparabiles comites. Nam perpetrarent, ut ista cura esset, lautarum facultas epularum. Ego autem tenui victus et cibis vulgaribus vitam egi letius quam cum exquisitissimis dapibus omnes Apicii successores. Convivia que dicuntur — cum sint comessationes modestie et bonis moribus inimice- semper michi displicuerunt. Laboriusum et inutile ratus sum ad hunc finem vocare alios nec minus ab aliis vocari. Convivere autem modeste cum amicis adeo iocundum, ut eorum superventu nil gratius habuerim nec unquam volens sino sotio cibum sumpserim». Cito dal testo critico fornito da Karl Enenkel, in: Karl Enenkel, Betsy De Jong-Crane e Peter Liebregts (a cura di), Modelling the Individual. Biography and Portrait in the Renaissance. With a Critical Edition of Petrarch's «Letter to Posterity", Amsterdam-Atlanta: Rodopi, 1998, p. 243-281 (p. 258). 
Il disegno comincia a prendere forma (e sarebbe immediatamente confermabile con altre facili citazioni di carattere autobiografico, per esempio Fam. VIII 4, 1-6; XIX 16, 19-21; XXI 13, 5, ecc.) e il discorso sulle abitudini alimentari trova la sua giusta collocazione entro una serie di dicotomie strutturali che emergono appena si rilegga il passo: ricchezza $v s$ mediocrità; affanni $v s$ serenità; cibi elaborati e abbondanti $v s$ cibi semplici e contenuti; banchetti smodati e volgari $v s$ cene frugali che proprio per questo sono occasione di colloquio affettuoso con pochi e scelti amici (né impediscono la riflessione e persino la creazione, visto che sulla mensa di Petrarca era sempre pronto, tra i semplici cibi, anche l'occorrente per scrivere, teste Fam. XXI 12, 26: «tra le rustiche portate spicca sempre la penna, e non mi si apparecchia mai la mensa senza che vi siano fogli per scrivere»). ${ }^{5}$ Alla «tavola» dei disvalori correnti e dei pessimi comportamenti che da essi derivano si contrappone la tavola dei valori che il personaggio Petrarca incarna e di cui dà quotidiana testimonianza attraverso concreti modi di vita. Anche l'atto del cibarsi, dunque, e l'insieme delle sue scelte alimentari contribuiscono a definirne la personalità e si armonizzano e istituiscono una rete di corrispondenze significative con altri atti e altre scelte.

Così, saltando una serie di possibili sviluppi del discorso (per esempio, il passo appena citato è puntualmente ripreso e sviluppato nel De remediis I 19, De conviviis, $₫ 6$, ove torna la stessa distinzione tra comessatio e convivium), il pensiero va alle celebri pagine che sono all'inizio del De vita solitaria. Qui Petrarca con immediata ed efficace forza didattica anticipa i contenuti essenziali della sua dimostrazione contrapponendo attraverso l'intero arco della giornata la figura dell'occupatus a quella di un solitarius ch'è la trasparente, idealizzata proiezione di se stesso, come sarà evidente alla fine del libro I, 9, 35, dove quella contrapposizione è riassuntivamente riproposta e dove il solitarius coincide, appunto, con l'autore: «So di un uomo [...] che ha scelto di vivere in solitudine, contento di poveri cibi e degli studi che gli appartengono", ecc. ${ }^{6}$ Lattacco è diretto:

5. «semper calamus agrestes inter epulas eminet, nec ulla michi mensa sine pugillaribus tabellis instruitur» (letteralmente, dunque, non fogli, ma «tavolette cerate»).

6. «Scio hominem [...] in solitudine constitutum, agresti victu et studiis contentum suis». Cito da F. P., De vita solitaria. Buch I, kritische Textausgabe und ideengeschichtlicher Kommentar von Karl A. Enenkel, Leiden: Brill, 1990, p. 120. Da qui anche le citazioni che seguono, dal cap. 2 dello stesso primo libro, p. 64-74. Che Petrarca voglia far trasparire se stesso attraverso il suo solitarius è del resto evidente se, tra tante altre cose, si rilegge la bella parte dedicata alle giornate valchiusane così come sono rievocate nella famosa Senile X 2 de mutatione temporum, a Guido Sette (Prose, p. 1104-1106), da leggere nella recente edizione: P., Lettres de la vieillesse. Édition critique d'Elvira Nota. Trad. de Claude Laurens. Présentation, notices et notes de Ugo Dotti, mises en français par Frank La Brasca, Paris: Les Belles Lettres, 2004, t. III, p. 240-273 (in part., p. 252-254, \$\$ 23-27 = p. 1104-1106 nell'edizione delle Prose ricciardiane, Milano-Napoli, 1955). Va almeno ricordato che tra i lontani ma sicuri archetipi di questo capitolo petrarchesco sta Luca 16, 19-31, e, precisamente per la figura dell'occupatus, SENECA, De brevitate vitae, passim (ma vedi in part. i capitoli 10-13). Su questa parte del De vita solitaria si legga ora, anche se in chiave diversa dalla mia, Ilaria TUFANO, «La notte, la paura, il peccato. Il ritratto dell" «occupatus» nel De vita solitaria», Rassegna europea di letteratura italiana, 22, 2003, p. 37-52. 
Ma che bisogno c'è di molte parole? Prendiamo di petto l'argomento e manteniamo le promesse. Mettiti sotto gli occhi della mente i due uomini di opposte abitudini che ti descriverò, e quello che vedrai in essi fa conto che valga per tutti gli altri. Nel mezzo della notte si alza l'occupato, infelice abitante delle città...7

L'occupatus si alza, dunque, atterrito da notturne visioni, e sùbito s’immerge nella complessa trama delle turpitudini e degli inganni ai quali dedicherà l'imminente giornata, mentre il solitarius si alza felice al canto dell'usignolo e innalza commosso la sua lode a Dio:

nessuna voluttà degli occupati, nessuna delizia cittadina, nessun lusso regale può eguagliare la sua condizione. Contemplando il cielo stellato e anelando con tutto il suo cuore al Signore Iddio che lo abita, e pensando, nel luogo dell'esilio, alla sua vera patria, sùbito si dedica allo studio attraverso letture tanto istruttive quanto piacevoli, e così nutrito di cibi deliziosi attende l'imminente inizio del giorno con perfetta pace dell'animo. ${ }^{8}$

I cibi deliziosi sono quelli che la lettura somministra, naturalmente (vedine un altro bell'esempio in Fam. XVII 1, 1), ma il cibo metaforico non tarda a trovare corrispondenza nel cibo reale, che finisce per essere l'elemento concreto, visivamente e persino drammaticamente concreto, che marca la differenza tra i due. Mentre i crimini dell'uno e l'esemplare vita dell'altro sono riassuntivamente riferiti, il modo di mangiare dell'occupatus è descritto in presa diretta, e i suoi pranzi segnano i momenti culminanti della giornata e ne condensano i contenuti. E l'efficacia delle opposte rappresentazioni vale, appunto, più di tante parole. Il pranzo del potente è il trionfo della confusione, del lusso, dello spreco, della sporcizia, in un impressionante e caotico crescendo che vede andare e venire complicati cibi dagli improbabili colori e odori e sapori, sospetti addirittura di veleno, mentre il padrone di casa affonda tra i cuscini pallido e gonfio per le gozzoviglie della sera precedente e tutto preso dal pensiero dei suoi intrighi: e suda, puzza, rutta, sbadiglia, e mangiucchia con aria schifata ora una cosa ora l'altra.' E quando, ore dopo, se ne va barcollante tra servi e parassiti

7. «Sed quid opus est multis? Agamus ipsam rem et, quod promittimus, persolvamus; duos homines contrariis moribus, quos tibi describam, ante tue mentis oculos pone et, quod in illis vides, in cuntis existima. Surgit occupatus, infelix habitator urbium, nocte media»...

8. «quem statum nulla voluptas occupatorum, nulle unquam urbane delitie, nulli regnorum fastus equaverint. Inde suspiciens celum ac stellas et illic habitantem Dominum Deum suum tota mente suspirans et patriam cogitans de exilii sui loco, protinus ad honeste cuiuspiam iocundeque lectionis studium convertitur; atque ita cibis pastus amenissimi multa cum pace animi venture lucis initium prestolatur».

9. «llle sub ingenti et ruinam minitante aula componitur pulvinaribus obrutus sepultusque [...] obducta fronte, gravibus oculis, supercilio umbroso, rugosa nare genisque pallentibus, egre viscosa labra disglutinans, vix caput attollens, fulgoribusque et odoribus attonitus. Ubi sit nescit, vespertina tumens crapula et matutini negotii confusus eventu futurisque iam fallaciis intentus - Quo se vertat, aut quid agat? — sudat, olet, ructat, oscitat singula morsitans, universa fastidiens». 
ubriachi, lascia una sala puzzolente, sudicia, dal pavimento sdrucciolevole e schiumoso per le salse e il vino versato e per gli spruzzi di tiepido e viscido grasso, pieno di ossa e di resti ancora sanguinolenti, quasi sia non una sala da pranzo ma la stanza di un carnefice. ${ }^{10} \mathrm{E}$ poche ore dopo, al calar della notte, tutto si ripete in una decomposta, corrotta dimensione funeraria che aggiunge orrori e colori a uno spunto già di Seneca (è il motivo del ventris oboediens come morto vivente che ha fatto della casa il proprio sepolcro, in Ad Luc. 60, 4):

Ecco torna la notte, e quello torna alle gozzoviglie, con grandissima pompa e una lunga fila di persone avanti e dietro. Lo crederesti il funerale di un vivo, preceduto dai flauti affinché nulla manchi alla cermonia, mentre il sontuosissimo cadavere cosparso di preziosi profumi è di nuovo sepolto tra i cuscini quand'è ancora tiepido e ancora respira: poi, aggiungendo a un pranzo indigesto una cena pesante, prepara la nausea per il giorno seguente e ingombra la via al pranzo che verrà. ${ }^{11}$

Ho citato molto, ma forse non abbastanza: passo passo occorre infatti mantener presente l'opposto ritratto del solitarius che frugalmente si ciba nel nitore e nella pulizia della sua rustica dimora, e s'immerge e gode nell'intatta natura che lo circonda e ripete a sera, «solo, silenzioso, sereno, simile a un angelo» le sue preghiere, ${ }^{12}$ disponendosi al sonno non solo con animo più lieto, ma anche con un corpo più sano e più efficiente, dal momento che proprio la moderazione giova alla salute, mentre chi più concede alle voglie del corpo più lo danneggia. ${ }^{13}$

10. «Aule quidem insuavis odor, inamenus color, iter incertum, solum omne salsamentis effusis late olidum cruentumque, idem et vino lubricum, et fumo nubilum et spumis horridum et aspergine tepidum et adipe tabidum et ossibus albicans et sanguine rutilum». A proposito di un siffatto quadro, si veda nel capitolo sulla gola, in Carla CASAGRANDE e Silvana VeCCHIO, I sette vizi capitali. Storia dei peccati nel Medioevo, Torino: Einaudi, 2000, p. 145, la citazione da Vincenzo di Beauvais contro il modo disgustoso e indecente di comportarsi a tavola, da suo De eruditione filiorum nobilium (a cura di Arpad Steiner), Cambridge Mass.: The Medieval Academy of America, 1938, p. 121-122.

11. "Ecce redit nox. Ille redit ad crapulam: pompa ingens, ante retrorque longum agmen; vivi hominis funus putes: precedunt funalia et tybie, ne quid desit exequiis, et sumptuosissimum cadaver, perfusum caris odoribus, rursus inter pulvinaria sepelitur —adhuc tepidum, adhuc spirans. Inde gravem cenam indigesto superaddens prandio venture lucis nauseam parat et alteri prandio precludit iter»

12. Nell'àmbito di quello che è un vero e proprio topos contro la golosità dei monaci e i loro banchetti, la CASAGRANDE e la VECCHIO, op. cit., p. 140-141, citano un bel passo di Bernardo di Chiaravalle, dall'Apologia ad Guillelmum abatem (in Opera [...], Roma: Editiones Cirstercienses, 1963, III p. 97-98: qui purtroppo devo ometterlo, come tanti altri, per ragioni di spazio) che per molti aspetti fa il paio con questi di Petrarca. Se poi aggiungiamo quanto lo stesso Bernardo diffusamente scrive a proposito della "cena di Cristo", Sermones dominicales 29, 9, prendendo spunto da Luca 14, 16: «Homo quidam fecit cenam magnam et vocavit multos», avremo un dittico composto dalle due "cene», la terrena e la celeste, che sta sicuramente a monte della descrizione di Petrarca.

13. «Neque solum letiore animo, sed saniore etiam corpore membrorumque ministerio promptiore utitur; constat enim animi virtutes, precipueque modestiam, sanitati multum conferre corporee et frequenter, qui plus corpori serviunt, plus obesse». 
In queste pagine, Petrarca raggiunge uno dei risultati più notevoli della sua abilità retorica e della sua forza argomentativa e, per quanto più ci interessa, moltiplica quelle contrapposte tavole dei disvalori e dei valori, e via via le specifica all'interno di una cornice generale che provvisoriamente potremmo definire come: barbarie vs civiltà, ove la barbarie apparirebbe essenzialmente come un insieme di mortiferi comportamenti "contro natura», e la civiltà, per contro, come qualcosa di intrinsecamente vitale e naturale. Lasciando da parte gli specifici comportamenti criminali dell'occupatus e le virtuose pratiche del solitarius, la frugalità e la semplicità di pochi e rustici cibi continua ad opporsi all'abbondanza e all'artificiosità di piatti pesanti e indigesti, ma ciò comporta pure il contrasto tra lusso e mediocrità, tra spreco ed economia, tra eccesso e moderazione, tra sporcizia e pulizia, tra malattia e salute ... A rileggere tutto il lungo passo non sarebbe difficile continuare, né si può scansare la più che fondata impressione che Petrarca, anche sulla sola base della forza straordinaria di questo capitolo, meriterebbe finalmente un posto di primo piano tra i fondatori della «civiltà delle buone maniere», per dirla con il titolo italiano di un libro famoso. ${ }^{14}$ Sul punto dovrò brevemente tornare, ma al momento basti aver fissato che le opposte serie si dispongono in modo perfettamente concatenato e simmetrico, sì che la vita cittadina si contrappone alla vita solitaria; la corruzione, la disonestà e la violenza a una vita pura e incontaminata; il lusso sfacciato e volgare allo stile e all'eleganza della misura; il cibo artefatto ed eccessivo alla dieta fatta di cose semplici e naturali; le malattie per stravizi alla salute per sobrietà. E che da una parte sola, tutte assieme, stanno la vita cittadina, la corruzione, il lusso, l'eccesso di cibi, la malattia, e dall'altra la vita solitaria, l'onestà, la mediocrità, la dieta e la buona salute. ${ }^{15}$

Questo non è, naturalmente, che uno schema assai sommario che tralascia molte cose importanti e mira soprattutto a quel discorso sul cibo che mostra d'avere una parte non secondaria nel sistema di Petrarca, sulla base dei testi sin qui citati e su molti altri che si dovranno considerare, sino al relativamente tardo De remediis e alle importanti e belle Seniles V 3 al Boccaccio; XII 1 e 2 al Dondi; XV 3 a Lombardo della Seta; XVI 3 a Francesco da Siena. Nei quali, sia detto sùbito, troviamo una ininterrotta serie di conferme (e di interessan-

14. Norbert Elias, La civiltà delle buone maniere. La trasformazione dei costumi nel mondo aristocratico occidentale [1969], Bologna: il Mulino, 1982 (in originale: Über den Prozess der Zivilization. I. Wandlungen des Verhaltens in den Weltichen Oberschichten des Abendlandes).

15. Un esempio di simili opposizioni, che ripete l'invito al lettore che è nel De vita solitaria, è nella Fam. X 3, diretta al fratello Gherardo, $\$ 42$ : «Paragona, ti prego, la tua condizione presente con la passata: la povertà sicurissima con le rischiose ricchezze, i dolci riposi con l'amarezza delle preoccupazioni, gli eccellenti confratelli con i nemici malvagi, e ancora il silenzio con le risse, la solitudine con le folle, le selve con la città, i digiuni con le gozzoviglie ...» [Confer, oro, nunc ista cum illis: cum turbulentis opibus quietissimam paupertatem, cum amaris negotiis dulce otium, cum iniquis hostibus optimos fratres, denique cum litibus silentium, cum turbis solitudinem, cum urbibus silva, cum commessationibus ieiunia ...], ecc. 
ti corollari) rispetto a quanto è emerso sin qui. In particolare, lasciando l'aspetto negativo della questione, vorrei soffermarmi un attimo su due delle connessioni significative, e generatrici a loro volta di una rete di connessioni ulteriori, che Petrarca istituisce: quella tra la frugalità e la vita solitaria, e quella tra la frugalità, se non proprio l'astinenza, e la salute.

\section{II}

L'intima connessione tra la vita solitaria e una dieta semplice e sana è un dato assolutamente conclamato ed evidente nel De vita solitaria, in termini tali che non resterebbe che prenderne atto, senza troppe elucubrazioni, così come appare del tutto scontato, di per sé, l'elogio universalmente topico della frugalità, che del resto campeggia sin dalle prime righe del Regimen sanitatis salernitano (se vuoi vivere sano, bevi e mangia poco: "parce mero, coenato parum»). ${ }^{16} \mathrm{Ma}$, come sempre, la cosa ha articolazioni più ricche, per la semplice ragione che essa rientra altrettanto chiaramente in un quadro che mira a tracciare un intero modello o progetto di vita in cui tout se tient, dalla più umile e concreta quotidianità alle scelte morali più ardue, e che questo quadro è precisamente quello in cui Petrarca inserisce, ora in maniera indiretta e ora in maniera più sottile e obliqua, se stesso. Di tale quadro, insomma, egli è insieme autore e parte, sì che non è affatto esagerato considerarlo una sorta di autoritratto ideale, minuziosamente percorso dalle sue personali idiosincrasie e strategie che hanno nell'elaborato mito della "vita solitaria» uno dei punti di riferimento più solidi e costanti. Qui non tento neppure di entrare nel merito di tale mito, perfetta espansione sul piano etico di quel mito di Valchiusa al quale Petrarca ha affidato tanta parte della sua identità di uomo e scrittore, e m'accontento invece di illustrare meglio come dei tanti e complessi aspetti che lo compongono faccia parte, modesto quanto si voglia ma assolutamente coerente con il tutto e infine necessario, anche quello che riguarda il cibo. Né in effetti si potrebbe immaginare una più compiuta opposizione di quella che distingue la corrotta vita cittadina e le tristi e sozze gozzoviglie dell'occupatus dall'agreste semplicità della «munda mensa» del solitarius: un'opposizione che specializza, per dir così, quella più generale secondo la quale l'infernale Avignone/Babilonia rischia addirittura di contaminare con il suo fetore persino l'innocente purezza, l'innocuam puritatem dell'appartata Valchiusa (Fam. XIII 8, 16). Del resto, seppur fuori da contesti violentemente polemici, Agostino riassume con efficacia nel II del Secretum, nel passo de gula:

Non mi pare il caso di parlare del vizio della gola, del quale sei affatto libero, se non fosse per qualche banchetto un po' più ricco con il quale talvolta asse-

16. Ma è anche fondamentale eliminare le preoccupazioni, e non adirarsi: «Si vis te vita vivere sanum, / curas tolles graves, irasci crede profanum, / parce mero, cœnato parum ...» (Flos medicinae Scholae Salerni 2-4, in Collectio Salernitana ossia Documenti inediti e trattati di medicina [...], pubblicati a cura di Salvatore De Renzi, Napoli: Tipografia del Filiatre-Sebezio, 1852 , I p. 445). 
condi il piacere degli amici. Ma da questa parte non temo nulla: infatti, tutte le volte che la campagna strappa il suo uomo alle città e se ne riappropria, immediatamente spariscono tutti i rischi di siffatti piaceri. ${ }^{17}$

I rischi di una sia pur relativa concessione al cibo spariscono ogni volta che Valchiusa recupera il suo fedele, amante di cibi agresti a base di ortaggi, e solo raramente disposto a qualcosa di più, come egli diffusamente spiega anche in Fam. VIII 4, 3-6: «tu sai che a me piacciono povere mense e cibi contadini, e che almeno nella moderazione nel mangiare vado d'accordo con Epicuro, secondo il quale il massimo della voluttà ch' egli tanto loda sta nelle verdure dell'orto [...] non ch'io rifiuti qualcosa di più ghiotto, purché mi si presenti assai raramente, e sia passato molto tempo tra una volta e l'altra». ${ }^{18} \mathrm{E}$ ciò si fa ancor più evidente nel momento in cui Petrarca invita qualcuno nel suo ritiro, offrendo un programma di perfezione bucolica che comprende ogni volta un cibo che direi moralmente e stilisticamente adeguato, proprio quale vuol presentarsi il "pranzo poetico» al quale è invitato, presso la sorgente del Sorga, Angelo Acciaiuoli (Fam. XII 11, 10). Ed è altrettanto adeguata all'ambiente e alle scelte di vita del protagonista la cena levis alla quale è condimento l'appetito, la fatica e il digiuno di tutto il giorno nella bellissima Epyst. I 6, 158 s., ove il quadro idillico delle giornate valchiusane offre una versione addolcita e personale, ma nella sostanza altrettanto radicale, di quella stessa opposizione che divideva l'occupatus e il solitarius. Così nell' Epyst. III 1, 90 s., a Giovanni Colonna, per esempio, già citata in apertura, egli vorrebbe mostrare al cardinale come abbia munito di massi il suo piccolo campo per proteggerlo dalla forza del fiume in piena:

Orsù dunque, vieni a vedere, se hai voglia di una quiete come la mia. Ti renderai conto di preferire questi ozi alle guerre cittadine, e non aver paura dei rustici cibi e dei rozzi letti di rigida paglia [...] ti offrirò i succosi grappoli di questi colli pieni di viti e fichi dolcissimi e acqua fresca appena attinta dal cuore della sorgente. ${ }^{19}$

17. «Gule nulla fit mentio, cuius studio nullatenus tenereris nisi, voluptati favens nonnunquam amicorum, blandior convictus obreperet. Veruntamen nichil hinc metuo: quotiens enim urbibus ereptum rus suum recuperavit incolam, omnes repente diffugiunt insidie talium voluptatum» (F. P., Secretum, [a cura di Enrico Fenzi], Milano: Mursia, 1992, p. 168). Ricordo che per quanto riguarda se stesso, Agostino dichiara di domare i desideri del corpo con il digiuno, e di assumere il cibo al puro scopo della sopravvivenza, come fosse una medicina (Conf. X 31, 43).

18. «scis me rustico apparatu et cibis agrestibus delectari, et in tenui victu solum cum Epycuro sentire, cui in ortulis et oleribus illius a se laudate voluptatis summa reponitur [...] lautiora non respuam, sed que rarissime et magno redeant intervallo». L'esempio di Epicuro è ricordato ancora in De remediis II 10, 16, e in Sen. XV 3 (nell'Opera omnia di Basilea, 1554, p. 1033), sulla scorta di SeneCA, Ad Luc. 18, 9; Cicerone, Tusc. V 89; GiovenaLE, XIII 122-123.

19. «Hec, age, visurus propera, par siqua quietis / cura tibi; cernes bellis hec otia quantum / urbanis preferre velis; neu dura timeto / fercula et horridulis inculta cubilia culmis [...] nos tibi pampineos colles gravidosque racemos denique mellifluas ficus undamque recentem / gurgite de medio offerimus...» 
E ancora invita lo stesso Colonna nell' Epyst. III 4, 57-66, a raggiungerlo a Valchiusa, non più per fargli contemplare un'opera che le acque sono tornate di nuovo a travolgere, ma piuttosto per dimostrargli come egli si sia adeguato alla natura, e si sia trasformato in appassionato pescatore:

Mie armi sono ora le reti e i labirintici percorsi delle nasse intrecciate di vimini che lasciano entrare l'acqua e si trasformano in una prigione dalla quale $\mathrm{i}$ pesci non riescono in alcun modo a uscire. Diventato pescatore, invece delle spade uso ami ricurvi ricoperti di fallaci esche, e aste flessibili, e un piccolo tridente con il quale già ho imparato a immobilizzare i pesci colpendoli sul dorso e a inchiodarli sui sassi del fondo. Ecco, ti mando queste primizie della mia arte fluviale, e tanti versi quante case ha la piccola Valchiusa che ti riserva piccoli pesci e rustici carmi. ${ }^{20}$

Il gioco, contro ogni apparenza, è assai sottile, e sottilmente (ma non troppo, forse) polemico. Petrarca esibisce infatti un'identità e un modello di vita alternativi rispetto al mondo che il suo patrono e protettore autorevolmente rappresenta, sino a trasformarsi in tutt'altra persona, piscator factus, che rovescia i tortuosi e pericolosi percorsi della vita di corte, là, ad Avignone, in altrettante scelte di potere e di libertà, e ne rivendica con orgoglio la dimensione separata e totalizzante. Egli evita volutamente di nominare la carne (torneremo sul punto), e cioè il cibo dei signori, e si presenta come un pescatore e si ciba di pesciolini, così come, nella Fam. XIII 8, nello stesso contesto dal quale ho tratto la citazione d'apertura, si veste in modo tale da sembrare un contadino o un pastore $(\$ 10$ : "Che dirò dei vestiti, e delle scarpe? [...] Mi prenderesti per un contadino, o un pastore»), ${ }^{21}$ e insieme lascia il pane bianco che i suoi servi gli portano e si ciba con gusto dello stesso pane della sua vecchia contadina, un pane che sa e ha il colore della terra, prope terreus panis, replica di quello mucidus e lapidosus della Fam. III 13, 2, e di quello duro e mal fatto e composto di cereali diversi, durum et inelaboratum atque ex omni fruge compositum raccomandato nella Fam. XIII 4, 24:22 e l'affermazione trae particolare significato dal fatto che, come ben si sa, il pane bianco di frumento era e resterà a lungo un consumo cittadino e di lusso ancora per secoli, mentre il pane dei contadini era fatto di cereali poveri come orzo, miglio, avena, segala, panìco, sorgo. ${ }^{23}$ Qui e là, insomma, nessun particolare è irrelato rispetto al program-

20. «Retia nunc sunt arma michi et labyrithius error / viminea contextus acu, qui pervius undis / piscibus est carcer nulla remeabilis arte. /Pro gladiis curvos hamos fallacibus escis / implicitos tremulasque sudes parvumque tridentem / piscator modo factus ago, quo terga natantum / sistere iam didici duroque affigere saxo. / Primitias en fluminee transmittimus artis / et versus quot clausa domos habet arctaque vallis, / que tibi pisciculos et rustica carmina pascit».

21. "Quid de vestibus, quid de calceis loquar? [...] Agricolam me seu pastorem dixeris».

22. Anche questo motivo è già in SENECA, Ad Luc. 123, 2.

23. Un ricco panorama in Massimo MONTANARI, Alimentazione e cultura nel Medioevo, Bari: Laterza, 2004 (prima ed., 1988), nel cap. VII, "Modelli di civiltà: il consumo di cereali», p. 124-146. Confermano che il pane bianco resta a lungo un consumo di lusso le minuziose schedature di Ingemar Boström: cfr. Anonimo Meridionale, Due libri di cucina (a cura di 
ma di fondo, ancorato alla esplorazione di una dicotomia che non solo oppone, diremmo per comodità, Avignone a Valchiusa, ma assume i caratteri di una personale scelta morale dalle larghe valenze politiche, e dunque di un prima e di un poi ancorato al tempo di una mutatio interiore: «la ragione del cambiamento sta semplicemente nel fatto che oggi soprattutto mi spiace quello che in passato soprattutto mi piaceva [...] ora, ai miei occhi in nessun modo più mi piaccio che libero e indipendente». ${ }^{24} \mathrm{La}$ frugalità, insomma, è intrinseca a una scelta di vita che mira a sciogliere legami servili, e va in cerca della libertà e della indipendenza personale, quasi realizzando antichi precetti: «Diogene lavava della verdura, e Aristippo gli disse: "Se tu accettassi di adulare Dionigi, non dovresti mangiare questa roba". E Diogene rispose: "Se tu, invece, mangiassi questa roba, non dovresti adulare Dionigi" ». ${ }^{25}$ Né irrelata è, dunque, la maschera piscatoria di cui la libertà del solitarius si riveste, e in definitiva i pisciculi stessi, forti di un loro specialissimo statuto, visto che in essi potremmo compendiare, con poche altre cose, sia la frugalità classica che quella cristiana. Augusto, infatti, a quanto ne dice Svetonio direttamente citato da Petrarca in Fam. XXII 14, 22 e in De remediis I 18, 6, si accontentava di un cibo assai scarso e comune: soprattutto preferiva pane raffermo, piccoli pesci, formaggio di vacca pressato a mano e fichi vernini' ${ }^{26}$ E Girolamo, per parte sua, non solo scriveva nell'Epistula ad Paulinum: "Cibati poveramente e di sera, e abbiti quali massime delizie le vedure, i legumi e ogni tanto qualche pesciolino» (non sarà che Petrarca si ricordi proprio di lui nella citazione già fatta, da Fam. XIII 8, 9, che in latino suona, ripeto: «Uva ficus nuces amygdale delitie mee sunt; quibus hic fluvius abundat, pisciculis delector»?), ma dava anch'egli colore in senso lato politico alla scelta della frugalità, proprio come nell'aneddoto di Valerio Massimo: «Dove si mangiano verdure da poco prezzo e rustico pane e si usa con moderazione del cibo e delle bevande, lì le ricchezze non servono né ha luogo l'adulazione, che non mira ad altro che all'interesse». ${ }^{27}$

Si direbbero, questi, i modelli più precisi ai quali Petrarca ricorre, ma in verità l'arco delle «fonti» è talmente ricco e legato a una topica tanto consoli-

I. Boström), Stockholm: Almqvist \& Wiksell International [Acta Universitatis Stockholmiensis - Romanica Stockholmiensia 11], 1985, p. 68-69 e 76 (ma vedi già Ferdinad BRAUDEL, Civilisation matérielle, economie et capitalisme, Xve-XVIII siècle. I: Les structures du quotidien: le possible et l'impossible, Paris: Colin, 1979, I, p. 110-111). Ma il pensiero va naturalmente ai quadri terrificanti di vita contadina rievocati da Piero CAMPORESI, Il pane selvaggio [1980], Milano: Garzanti, 2004.

24. ibid. \$\$ 10-11: «et mutati habitus nulla sit causa nisi quia quod primum placuit primum sordet $[\ldots]$ meis autem oculis nullo modo magis placeo quam solutus ac liber».

25. Valerio MASSIMO, IV 2 ext. 4.

26. Aug. 76: «Cibi [...] minimi erat atque vulgaris fere. Secundarium panem et pisciculos minutos et caseum bubulum manu pressum et ficus virides biferas maxime appetebat».

27. Epist. 58, 6: «Sit vilis et vespertinus cibus; olera et legumina interdumque pisciculos pro summis ducas delitiis»; Epist. 79, 4: «Ubi vile olusculum et panis cibarius et cibus potusque moderatus, ibi divicie supervacue, ibi nulla adulatio, que precipue fructum respicit». Vedi IsIDORO, Etym. XX 2, 15: «Cibario è il cibo grossolano che si dà ai servi per loro sostentamento" [Cibarius est qui ad cibum servis datur, nec delicatus]. 
data che non si saprebbe letteralmente donde cominciare: per esempio, un discorso sulle sue classiche auctoritates sarebbe forse semplice ma anche nello stesso tempo amplissimo, dovendo per forza investire non solo lo specifico àmbito dei cibi ma l'intero mondo morale connesso ad una antica ed eroica $\mathrm{e}$ infine stoica frugalità di matrice "repubblicana» e alla nefasta dimensione sociale del binomio lusso-gola, eloquentemente denunciata da Svetonio, in particolare là dove parla di Giulio Cesare e di Augusto, e da Cicerone, soprattutto Tusc. V 89 s., e da Orazio, naturalmente, e da Seneca e Giovenale per il forte coté polemico... Ma prenderemmo, così, altre vie, rispetto a una prima ricognizione del solido sistema costruito da Petrarca. Il quale sui pesciolini insiste, per esempio invitando il Cabassoles a Valchiusa nel carme Exul ab Italia, ${ }^{28}$ nel quale torna ancora una volta ad elencare le delizie del luogo e istituisce la solita contrapposizione tra i malsani e lussuosi banchetti di città e i semplici e nutrienti cibi della sua campagna (v. 34: «il cibo serva al nutrimento, né si trasformi in portate che sono veleno per lo stomaco»), ${ }^{29}$ e infine, v. 49-54, invita pure l'amico a pescare con lui tra i sassi del Sorga, all'ombra degli alberi da frutto, e ancora gli promette pesche, mele e pere delle quali costui dovrà in ogni caso promuovere la coltivazione se vorrà avere un'arma in più per difendere la propria malferma salute, ora ch'è vecchio:

Dai rami carichi di frutti ci verrà un'ombra piacevolissima, mentre con gli ami ricurvi andremo frugando tra i massi rotondi. E Valchiusa ci darà il resto in abbondanza: e aggiungi pesche, mele, pere, delizie del dessert. Tu, ordina ai tuoi che te le procurino e non trattenerti dall'usarle come armi nei confronti della declinante vecchiaia ${ }^{30}$.

L'accenno al valore curativo della frutta direi che rende del tutto naturale il passaggio all'altro punto sul quale vorrei soffermarmi: quello che riguarda, in generale, la salute. Ma mi concedo ancora una sosta, piacevolissima per altro, su un testo che compendia con squisita leggerezza e intima serietà quanto sin qui s'è visto. Si tratta dell' Epyst. III 5, ancora a Giovanni Colonna, dedicata al grande cane (un molosso?), regalo del cardinale, che era diventato l'amico inseparabile delle solitarie giornate valchiusane di Petrarca. Ebbene, anche il cane, lasciato l'innaturale ambiente dei palazzi cittadini e i malsani e pigri lussi curiali, dopo un breve periodo di adattamento ha imparato a godere di quelle pure acque e di quella verzura:

28. Var. 3. Cito dall'edizione dei Poemata minora a cura di Domenico Rossetti, Milano: Società tipografica de' Classici italiani, II p. 60-66.

29. "cibus altor erit, stomachi non fercula pestis».

30. «Umbra ex pomiferis veniet gratissima ramis, /dum curvos scopulos uncis scrutabimur hamis. / Cetera clausa quidem Vallis prestabit abunde: / Persica, mala, pyra, mensae decus adde secundae. /Tu, precor, ista tuos iubeas perquirere, nec te / arma parum validae pigeat conferre senectae». Vedi OvIDIO, Met. IX 92: «tulit [...] et mensas, felicia poma, secundas». 
Già sta bene nei prati; già nuotando attraverso i limpidi fiumi ne morde l'acqua e impazza gioioso nei suoi puri gorghi. Già gli piacciono i miei cibi e la mia serenità senza problemi. Ha smesso ormai di preferire le ampie sale del padrone che ha lasciato e le sue variate vivande: pane e acqua gli bastano, e una modesta casetta. Lavate, splendono le membra del fiero animale, e la scabbia contratta nella pigrizia e nel torpore è caduta nella fonte salubre: ora porta più alta la testa, e rizza il collo irrobustito... ${ }^{31}$

Non c'è dubbio. Quello che fa bene al cane non è diverso da ciò che fa bene all'uomo. Anche al cane, infatti, pane e acqua sono sufficienti, come insegnava Seneca, Ad Luc. 24, 4 e 110, 18 (nella prima delle due lettere: «Nulla di ciò che abbiamo è veramente necessario: torniamo alle leggi di natura [...] la natura s'accontenta di pane e acqua, e rispetto a ciò nessuno è povero"), ${ }^{32}$ il cui insegnamento è riproposto da Agostino, nel l. II del Secretum, anche se con qualche polemica correzione moderatrice (sulla quale dovrò tornare più avanti) che non ne intacca la sostanza:

Non ti richiamo alle imposizioni di quelli che dicono: «Alla vita dell'uomo pane e acqua bastano: nessuno è povero rispetto a queste cose, e tutti quelli che avranno concentrato in esse i loro desideri sfideranno la felicità stessa di Giove». Né pongo dunque «il fiume e Cerere» a misura della vita umana. Queste sono infatti sentenze che suonano tanto magniloquenti quanto fastidiose agli orecchi dgli uomini, e da sempre sono odiate. ${ }^{33}$

$\mathrm{E}$ ad entrambi, contro la scabbia, giovano i bagni in acqua corrente, come ribadisce Epyst. III 25, 45-46: in Francia "c'è scarsità di sorgenti nelle quali possano ristorarsi i corpi malati», 34 e De remediis II 85, 8, ove si legge che la scabbia è una malattia ignobile che ha una nobile cura: lavoro, calore estivo, bagni, veglia, dieta. ${ }^{35}$ Con tutto ciò sarebbe forse esagerato dire che il cane è una "figura» di Petrarca: certo, anche l'animale finisce per anteporre alla servitù e ai sospetti lussi cittadini la sana e frugale libertà di Valchiusa, e la sua meta-

31. «Iam prata iuvant, iam lucida tranans / flumina mordet aquas luditque in gurgite puro; / fercula iam sibi nostra placent et libera curis / otia; deserti non ampla palatia regis / anteferat variasque dapes, nam panis et unda / sufficiunt ac parva domus. Iam membra refulgent / lota feri, cecidit scabies in fonte salubri / torpenti contracta situ, iam vertice toto / altior it solito cervixque torosior extat..."

32. «Nihil ex his, quae habemus, necessarium est: ad legem naturae revertamus [...] panem et aquam natura desiderat. Nemo ad haec pauper est». Ma vedi pure Lucano, IV 381, e, di Petrarca, ancora Sen. XI 3 (Opera omnia, p. 1033), e De remediis II 10, De tenui victu, $\$ 6$.

33. Secretum, p. 160: «Non igitur ad illorum statuta te revoco qui aiunt: "Satis est vite hominum panis et aqua; nemo ad hec pauper est, intra que quisquis desiderium suum clausit, cum ipso Iove de felicitate contenderet", nec modum vite hominum "fluvium Cereremque" [Lucano, IV 381] constituo. Sunt enim ut magnifice sic auribus hominum importune pridem odioseque sententie».

34. "scatebrisque caret quibus egra leventur corpora»: così l'ed. Rossetti, II, p. 72, ma non sarà piuttosto laventur?

35. "Morbus ignobilis sed nobilis cura est: labor, estas, balnea, pervigilium, dieta». 
morfosi, che comporta l'abbandono del vecchio padrone, ha indubbio valore di apologo ed è percorsa da precisi veleni polemici. Tant'è che la forte istanza di libertà e la riappropriazione dei più semplici e affatto naturali contenuti della propria vita ci rimandano ai rapporti dell'autore con il cardinal Colonna, e quasi appaiono una prefigurazione della rottura finale, nel 1347, alla quale dà voce definitiva l'egloga Divortium. Né a ciò s'oppone, nell'Epystola, quella sorta di goffa reprobatio finale, v. 99-105, e le vistosamente contraddittorie e posticce e infine false - una diabolica perfidia in più di Petrarca? - ultime parole: «il cane, se lo si lasciasse libero di scegliere, preferirebbe giustamente ritornare all'eccelsa Colonna». ${ }^{36}$ Quando invece, sullo sfondo, sembra di risentire Seneca là dove scriveva che un tempo lontano le capanne coperte di canne ospitavano uomini liberi mentre al presente nel marmo e nell'oro abita la schiavitù (Epist. 90, 10: "culmus liberos texit, sub marmore atque auro servitus habitat»).

\section{III}

Sia come sia, anche in questo ultimo caso, come ben si vede, il rapporto strettissimo tra la vita solitaria e la frugalità, all'insegna del rispetto delle leggi fondamentali della natura e sullo sfondo continuamente evocato di ciò che lo nega, raddoppia direi spontaneamente nell'altro: quello tra la frugalità e la buona salute. Rapporto che per la verità meglio si configura come un legame a senso unico, perché anche secondo Petrarca la frugalità è una delle condizioni, forse la più importante e certamente quella che è nel potere dell'uomo di controllare, della salute. Vediamo meglio, e aggiungiamo sùbito una precisazione ovvia ma necessaria. La frugalità della quale Petrarca parla ha due aspetti tra loro connessi: uno relativo alla quantità del cibo, l'altro relativo alla sua natura. Voglio dire che sarebbe astrattamente pensabile una frugalità che s'esercitasse nel consumo di fagiani e pernici e di vini pesanti e costosi, ma di fatto essa non esiste. Quel tipo di consumi di lusso è in ogni caso la deplorevole negazione della frugalità, che invece esige, per suo ferreo statuto, cibi semplici e naturali: in primo luogo ortaggi e frutta, come abbiamo visto, e l'acqua pura come bevanda. Restiamo alle raccomandazioni di Gerolamo e all'esempio canonico di Augusto, che vale per mille altri: la frugalità è insieme moderazione nella quantità e selezione nella qualità. La quale qualità riposa essenzialmente su due caratteri: quello comune e domestico del cibo - il cibo di tutti e alla portata di tutti, si direbbe- e quello che impone l'assenza di manipolazione e mescolanza e alterazione da parte dell'uomo (del cuoco, insomma, figura contro il quale Petrarca non manca di polemizzare). Se torniamo, infatti, ai cibi dell'occupatus, potremo facilmente constatare che la loro dominante negativa è costituita tanto dalla loro arrogante e costosa ricercatezza, quanto appunto dalla sistematica violenza che li maschera e stravolge sino a renderli irriconoscibili. Il vino, corretto con miele e succhi orientali e bacche profumate, muta per arte la sua natura; pesci e uccelli, ricoperti di rare spezie, 
non conservano della loro origine altro che il nome, e gli sbalorditivi piatti che vengono serviti sono il prodotto del capriccioso oltraggio dei cuochi. ${ }^{37}$ Tale è il tono dell'invettiva, da far pensare che Petrarca abbia direttamente avuto presente il De miseria humane conditionis di Innocenzo III, e in ispecie il cap. XVII, De gula, che muove dalla citazione di Ecli. 29, 28: "A fondamento della vita dell'uomo c'è acqua e il pane, e un vestito e una casa che evitino l'indecenza», ${ }^{38}$ e prosegue:

Ai golosi non bastano più i frutti degli alberi, i vari generi di legumi, le radici delle erbe, i pesci del mare, gli animali della terra, gli uccelli del cielo: ora, vanno in cerca di condimenti, si procurano spezie, allevano animali da prelevare ben grassi che saranno ingegnosamente cucinati dall'arte dai cuochi e lussuosamente imbanditi dai servi. Uno tritura e setaccia, un altro mescola e compone, e volge la sostanza in accidente e trasforma la natura in artificio, sì che la fame si sostituisca alla sazietà e la nausea sia respinta dal desiderio di stimolare la gola, non da quello di sostentare la propria natura. ${ }^{39}$

Ma probabilmente non solo questo ché, insieme ai passi di Bernardo di Chiaravalle già richiamati, molti altri stanno sullo sfondo, come mostrano le pagine del libro della Casagrande e della Vecchio. Per esempio, almeno questo che mi sembra specialmente pertinente, di Pietro il Venerabile:

Corrono da un luogo all'altro e volano rapidi come sparvieri o avvoltoi là dove vedono fumi di cucina o dove abbiano annusato profumo di carni arrostite e bruciacchiate [...] Le fave, il formaggio, le uova e persino i pesci sono venuti a noia: piacciono solo le pietanze degli egiziani. Il maiale arrostito o lessato, la giovenca grassa, il coniglio e la lepre, l'anatra scelta come la migliore dello stormo, le galline e insomma ogni quadrupede e volatile domestico ricoprono le mense dei santi monaci. Ma infine anche tutto ciò ha perso ogni valore: l'eccesso ha prodotto la nausea. Così, si è passati a delizie regali e stravaganti, e il monaco, sazio, non riesce a nutrirsi se non di caprioli, di cervi, di cinghiali e di orsi selvatici. ${ }^{40}$.

37. «Nec satis est nisi [...] naturam arte mutaverit»; «stupenda fercula et omne cocorum passa ludibrium».

38. "Initium vite hominis aqua et panis et vestimentum et domus protegens turpitudinem».

39. «Nunc autem gulosis non sufficiunt fructus arborum, non genera leguminum, non radices herbarum, non pisces maris, non bestie terre, non aves celi, sed queruntur pigmenta, comparantur aromata, nutriuntur altilia, capiuntur obesa que studiose coquuntur arte cocorum, que laute parantur officio ministrorum. Alius contundit et colat, alius confundit et conficit, substantiam vertit in accidens, naturam mutat in artem ut saturitas transeat in esuriem, ut fastidium revocet appetitum ad irritandam gulam, non ad sustentandam naturam». Cito da Lotharii Cardinalis (Innocenti III) De miseria humane conditionis (a cura di Michele Maccarrone), Lugano: Societas Tesauri Mundi [ma Padova: Antenore], 1955, p. 51-52.

40. Petrus Venerabilis, The Letters. Ed. with an Introduction and Notes by Giles Constable, Cambridge Mass.: Harvard University Press, 1967, I, p. 389: «Discurrunt de locis ad loca, et ut milvi aut vultures, ubi vel fumum coquinarum viderint, vel nidorem assae vel ustae carnis naribus hauserint, celeriter advolant [...] Faba, caseus, ova, ipsi etiam pisces, iam in nauseam versi sunt. Solae ollae Aegyptiorum placent. Assus aut elixus porcus, iuvenca pin- 
Ma su questa base, per dire così, Petrarca lavora di suo, splendidamente. E merita davvero citare:

se qualcuno, per quanto affamato, vedesse in quale maniera ripugnante e con quanto ruffianesco artificio siano stati messi insieme, se ne andrebbe saziato dal solo spettacolo. Vedrebbe infatti mescolati e reciprocamente ripugnanti cibi nostrani ed esotici, marini e terrestri, neri e candidi, aspri e dolci, pelosi e pennuti, domestici e feroci e, quasi sia rinnovato e concentrato in poco spazio quell'ovidiano Caos primordiale, non solo in un unico corpo ma in un unico vassoio «i corpi freddi combatteranno con i caldi, gli umidi con i secchi, i molli con i duri, i leggeri con i pesanti». E sotto un tale miscuglio di cose diverse e avverse, sotto tante vivande gialle e nere e livide, l'assaggiatore cerca con cura un veleno non a torto sospettato. ${ }^{41}$

Il passo è importante perché pone le basi negative per un possibile discorso dietetico che abbia come proprio fine la salute, e non la provocazione e la soddisfazione di ogni stimolo (si osservi di quanta ironia sia notata la ricerca del veleno in piatti che appaiono, di per sé, assolutamente velenosi!). Ma importante anche perché rinvia contemporaneamente alla teoria degli elementi naturali e delle loro combinazioni, e a una filosofia (nel caso, depravata) culinaria, che invece di essere rispettosa di quella teoria la stravolge. ${ }^{42} \mathrm{Al}$ proposito, può esse-

guis, cirogrillus et lepus, anser ex anserum grege electus, gallinae et prorsus omne quadrupes aut volatile domesticum, sanctorum monachorum mensas operiunt. Sed viluerunt iam et ista. Assiduitas multa fastidium ingessit. Ad regales et peregrinas delicias, transitus factus est, satur monachus iam non nisi caprea, cervo, apro vel urso agresti vesci potest». Si osservi come i cibi sùbito rifiutati siano quelli tipici del menu per religiosi (fave, formaggio, uova, pesce), mentre quelli finali sono tutti frutto della caccia, e cioè tipici dell'esclusivo menu dei nobili. Ancora, ho tradotto cirogrillus con "coniglio», ma il Du Cange, con esempi, dà chirogryllus come "coniglio", e cyrogrillus come "riccio", che andrebbe dunque altrettanto bene. A questa citazione Casagrande e Vecchio, op. cit., p. 141, ne fanno seguire un'altra altrettanto pertinente ed efficace di UGO DI SAN VITTORE, De institutione novitiorum XIX, (a cura di Hugo Feiss e Patrice Sicard), Brepols: Tournhout, 1997, p. 94-96.

41. «siquis valde licet esuriens cernat quam fede quantoque sint coagulata lenocinio, solo spectaculo satur abscedat. Ita mixta videbit invincemque certantia nostra exteris, marina terrestribus, nigra candidis, acria dulcibus, hirsuta pennatis, mansueta ferocibus et, quasi ovidianum chaos illud antiquum renovatum atque in angustias sit coactum, non solum corpore in uno, sed in una paropside, "frigida pugnabunt calidis, humentia siccis, / mollia cum duris, sine pondere habentia pondus" [Met. I 19-20: ma "pugnabant»]. Sub hac tanta colluvie diversarum simul et adversarum rerum, sub tot croceis atque atris liventibusque pulmentis, suspectum non immerito venenum sedulus pregustator explorat».

42. «I dati fisiologici della medicina antica e medievale si basano sulla teoria dei quattro umori, la cui origine risale al sistema filosofico di Empedocle e alle concezioni aristoteliche. Fondata sui quattro elementi, questa teoria precisa che ognuno di essi è retto da due qualità primarie, una attiva, l'altra passiva. Così il fuoco è caldo e secco, l'aria calda e umida, l'acqua fredda e umida e la terra fredda e secca. Ogni essere vivente è composto, in proporzioni variabili, di quattro umori, che sono essi stessi una mescolanza delle due qualità primarie: il sangue caldo e umido, la collera (o bile gialla) calda e secca, il flegma freddo e umido, e la melanconia [o bile nera] fredda e secca. La combinazione di questi umori definisce la complessione e il temperamento del soggetto. Nel corpo umano è così riprodotto 
re interessante osservare che, di là dalle fonti canoniche (per es. Seneca, Ad Luc. $110,13)$, qualcosa di analogo all'invettiva di Petrarca, che - lo vedremo- ben conosce quelle teorie, dice già Alano di Lilla quando, accusando soprattutto i prelati di canina aviditas per il cibo, denuncia precisamente come una violenza fatta alla natura l'eccesso di salse speziate nei cibi e la loro caotica, indiscriminata confusione: «Entro la stessa portata l'animale di terra è sommerso da un'inondazione di pepe, e nel pepe il pesce nuota e l'uccello è intrappolato nella stessa collosa salsa al pepe. E mentre tante specie diverse di animali finiscono imprigionate nel carcere di un unico ventre, l'animale acquatico e il terrestre e il volatile stupiscono nel vedersi tumulare nello stesso sepolcro». ${ }^{43}$

Ripigliamo in ogni caso dalla salute, che ha il suo fondamento primo e assoluto, come ho anticipato, nella frugalità, se non proprio nell'astinenza. Se è vero, e Petrarca per parte sua ne è certo, che poco basta alla sussistenza dell'uomo (il pane e l'acqua di cui parlava Seneca), ne deriva che ogni eccesso che oltrepassi quel limite naturale sia doppiamente rischioso: lo è sul piano morale, perché rappresenta un cedimento ai desideri del corpo e dunque alla brutalità dell'elemento materiale, a scapito delle superiori istanze della razionalità e insomma dello spirito. E lo è sul piano fisico perché, se l'eccesso diventa davvero tale e si radicalizza, è il corpo medesimo che ne soffre, essendo alterato l'equilibrio previsto dalla natura e artificiosamente forzate le sue necessità, sì che, come già abbiamo visto, è paradossalmente in nome delle supposte richieste del corpo che il corpo s'ammala (come abbiamo già ascoltato da Petrarca, spes-

il sistema dei quattro elementi. La salute risulta da un equilibrio degli umori in tutte le parti del corpo, mentre ogni squilibrio determina la malattia. Tuttavia, l'equilibrio perfetto è un puro dato teorico. Ogni individuo sano è caratterizzato, in realtà, dal dominio di un umore; l'equilibrio delle qualità è dunque relativo in rapporto all'assoluto, ma comunque armonioso. Si potrà così parlare di temperamento collerico (caldo e freddo), sanguigno (caldo e umido), melanconico (freddo e secco) o flemmatico (freddo e umido), ma anche di complessioni calde, fredde, secche o umide, quando è solo una delle qualità a dominare il corpo [...] L'alimentazione è una componente fondamentale della salute; ciò che si ingerisce, dopo diverse digestioni presentate come cotture, si trasforma in umori e si espande nelle diverse parti del corpo per formare gli organi, le membra o ancora le ossa. Ogni paziente sano deve mangiare alimenti che abbiano la sua stessa complessione; nel caso di una malattia, legata a uno squilibrio di umori, deve consumare cose contrarie per ristabilire l'equilibrio. Così un collerico mangerà alimenti caldi e secchi, ma per curare una malattia prenderà vivande fredde e umide. Alla base di questo sistema vi è un sistema di compensazione ...", ecc. Questo è il comodo e veloce riassunto di Marilyn Nicoud, La dietetica nel Medio Evo, nel vol. \& coquatur ponendo ... Cultura della cucina e della tavola in Europa tra medioevo ed età moderna. Coordinamento scientifico: Giampiero Nigro, Orazio Bagnasco, Angela Dillon Bussi, Allen J. Grieco, Bruno Laurioux, Marilyn Nicoud, Prato: Istituto int. di storia economica «Francesco Datini», 1998, p. 43-53 (p. 44-46). Ma vedi anche, sintetico e chiaro, il trattatello De quatuor humoribus ex quibus constat humanum corpus, nella Collectio Salernitana del De Renzi, cit., II, p. 411-412. Vedi avanti, nota 74.

43. «In eadem mensa terrestre animal piperis inundatione submergitur, piscis natat in pipere, avis eiusdem viscositate ligatur. Dumque tot animalium genera uno ventri ergastulantur in carcere, aquatile animal secum pedestre aeriumque genus in eodem sepulcro tumulari miratur» (Nikolaus M. HÄrING, «Alan of Lille, "De Planctu Naturae”», Studi Medievali, XIX, 1978 , p. $854=$ XII 11. 70-74). 
so nuocciono gravemente al corpo proprio quelli che più gli sono schiavi). Ora, è altrettanto importante osservare che in Petrarca questo aspetto puramente fisico della questione gode di autonomia propria, ed è dunque in grado di porre i propri fini e di definire un sistema razionale di cause ed effetti e regole che valgono per sé, indipendentemente dall'altro aspetto, quello immediatamente morale. La cosa è sottolineata, per non riferire che un caso particolarmente efficace (sostanzialmente già tutto contenuto, per altro, nel confronto tra l'occupatus e il solitarius), nel capitolo I 18 del De remediis, De lautu victu, $\$ 12$ :

Questo frugale regime alimentare rende gli uomi magri, efficienti e di bell'aspetto, senza alcun odore corporale che sia molesto a loro stessi e agli altri. Ora, metti a loro confronto quei ghiottoni mollicci, ansimanti, tremuli, puzzolenti che, per ripetere le parole di Cicerone, non fanno che sudare e ruttare: confrontali, e vedrai la differenza tra la frugalità e la crapula. Anche non fosse la virtù, basterebbe il loro aspetto a dirti quale sia la scelta giusta. E infatti non c'è nessuno, per quanto sia schiavo della gola, che dopo aver considerato a fondo la faccenda non debba ammettere che la frugalità sia di gran lunga preferibile. ${ }^{44}$

«Anche non fosse la virtù ...». Evidentemente, esiste più di una virtù, perché è pur sempre tale l'intrinseca razionalità che deve governare le diverse sfere dell'esperienza e che inevitabilmente approda, nel caso, all'ovvio riconoscimento sia dei danni prodotti all'organismo dal vizio della gola, stante il vecchio proverbio: "ne uccide più la gola che la spada» che Petrarca cita in Fam. III 10, 16: «quanti ne uccise la crapula o soffocò l'ubriachezza, che digiunando si sarebbero salvati! È in proverbio che ne uccide più il cibo che la spada, ${ }^{45}$

44. "Victus hic [tenuis et modicus] siccos et validos et aspectu placidos facit et odore corporis nec sibi nec aliis molesto: confer his madidos, estuantes, tremulos, olentes et, ut Ciceronis utar verbis, confer et sudantes et ructantes: tunc videbis quid inter frugalitatem intersit et crapulam. Etsi non virtus, et aspectus ipse monstrabit recta quonam vergat electio, ut vix sit quispiam tam gule servus qui acriter re discussa non sobrietatem longo illi preferendam fateatur». Il rimando è a CiCERONE, Tusc. V 99-100: «Aggiungi la snellezza e l'intatta salute che derivano da una simile moderazione alimentare, e paragonale con la condizione di quelli che sudano e ruttano rimpinzati di cibo come buoi all'ingrasso: capirai allora che proprio queli che più di tutti inseguono il piacere sono quelli che l'ottengono di meno, e che il piacere del mangiare sta nell'appetito, non nella sazietà» [Adde siccitatem, quae consequitur hanc continentiam in victu, adde integritatem valetudinis, confer sudantis ructantis refertos epulis tamquam opimos boves: tum intelleges, qui voluptatem maxime sequantur, eos minime consequi, iucunditatemque victus esse in desiderio, non in satietate]. Segue qui la citazione dalla lett. 7 di Platone, che dichiara di non amare chi si sazia due volte al giorno, che Petrarca riprende ben quattro volte, in De vita solitaria I 2, 12; De remediis II 10, 16; Sen. XII 1, 28, e XII 2, (Opera omnia, p. 1010).

45. «quam multos vel necavit crapula vel suffocavit ebrietas, qui ieiuni salvi forent! Vulgatum est plures cenam occidere quam gladium». In questa forma il proverbio parrebbe essere una variazione petrarchesca del «Ne uccide più la lingua che la spada», che deriva dal biblico Ecli. 28, 18. Per il senso generale, vedi fra tanti Ambrogio, De Cain at Abel I 5, 18: "molti ne ha uccisi la gola, nessuno la frugalità; a molti il vino ha fatto male, a nessuno l'astenersene» [ «Plurimos itaque gula sua occidit, nullum frugalitas: innumeris vina nocuerunt, nulli parsimonia»]; De Helia et ieiunio 4, 9: «il vizio della gola ci denuda, mentre digiunare serve a coprire anche gli ignudi» ["gula ergo nudos facit, ieiunia operiunt et exutos»]. 
sia dei vantaggi della moderazione che elimina appunto, con precisa corrispondenza, quei danni, com'è detto nel De remediis ancora, II 10, De tenui victu, $\$ 12$ :

Una siffatta frugalità caccerà la gotta da casa tua, eliminerà il mal di testa e le vertigini, il vomito, i rutti, la nausea, il sudore, e il malessere e la pesantezza che ti senti addosso, e l'alternanza di pallori e rossori, e gli odori sgradevoli della bocca e del corpo intero che infastidiscono sia te che gli altri. E ancora fermerà l'instablità del passo, il tremore delle mani, il dondolìo della testa e infine, ottima cosa, renderà più equilibrato e vigile il tuo spirito. ${ }^{46}$

L'elenco è dettagliato, e compendia bene quanto Petrarca ripete altrove in modo più occasionale. Di là dalle importantissime aperture all'aspetto estetico della faccenda, intimamente legato a quello della salute, ${ }^{47}$ già l'abbiamo sentito porre la dieta tra i rimedi della scabbia (De remediis II 85, 8). Lo stesso vale per la gotta, malattia da ricchi che si combatte con un'astinenza dal cibo, che può essere definita come una povertà volontaria e immaginaria, come spiega sia il De remediis II 84, 16, sia Fam. III 13, con il bell'apologo della podagra e del ragno nel quale Petrarca gioca di nuovo sulla minuziosa contrapposizione tra la giornata del povero e quella del ricco. ${ }^{48} \mathrm{Nel}$ De vita solitaria I $6,{ }^{49}$ ancora sulla base di Gerolamo, egli ricorda quei sacerdoti che con due o tre giorni di digiuno erano soliti eliminare gli umori del corpo generati dall'ozio e dall'immobilità, e conclude che in tal modo ne riusciva favorita la "divina» fertilità dell'ingegno. Nel De otio, cita la Bibbia, Ecli. 31, 22-24, a proposito dei malanni dell'intemperanza, mentre è la frugalità che assicura il «sonno della

46. «Hec tenuitas podagram tuis pellet e finibus, dolorem capitis auferet, cerebrique vertiginem vomitumque et ructum et nauseam et sudorem, tediumque et fastidium tui ipsius, pallorem alternum ac ruborem, odorem quoque et oris et corporis, tibi atque aliis importunum; sistet preterea pedes intabiles, manus tremulas, nutans caput, quodque est optimum, animum ipsum moderabitur frenabitque».

47. Il punto relativo alla dimensione estetica della buona salute meriterebbe uno sviluppo a sé. Ma si veda, intanto, anche per le numerose citazioni di testi, Danielle GourEVITCH, "L'esthétique médicale de Galien», Les études classiques, n. 55, 1987, p. 267-290; Véronique Boudon-MiLlot, "Médecine et esthétique: nature de la beauté et beauté de la nature chez Galien", Bull. de l'Association Guillaume Budé, n. 2, 2003, p. 77-91. Molti contributi sull'argomento anche nel $4^{\circ}$ Colloquio di Tours (Tours: CESR, 16 maggio 2003), Belles et vilaines maladies (a cura di Jean-Paul Pittion e Jacqueline Vons) (i riassunti, tra cui anche quello del citato saggio della Boudon-Millot, sono a disposizione sul sito www.cesr.univtours. fr).

48. "quam voluntariam seu fictam et imaginariam paupertatem dixerim» (De remediis, cit.). Una variante ulteriore, insomma, di quella tra occupatus e solitarius, nel De vita solitaria, che torna di nuovo, per esempio, in forme assai eloquenti, in Fam. XIII 4, 22-25, e nella Fam. VI 3, 50 (Gerolamo, Adv. Iovinianum II 13, PL XXIII col. 316, scrive che i podagrosi, una volta ricondotti a cibarsi in modo semplice e povero, guariscono: «ad simplicem mensam et pauperes cibos redactos convaluisse»).

49. Opere latine di F. P., (a cura di Antonietta Bufano, con la collaborazione di Basile Aracri e Clara Kraus Reggiani) (Intr. di Manlio Pastore Stocchi), Torino: UTET, 1975, I, rispettivamente, p. 336. 
salute», e, avanti, è ancora la victus parcitas che garantisce contro il potere insidioso e perturbante dell'attività onirica. ${ }^{50}$ Nella Disp. 46 (ma vedi anche il dettagliato resoconto in Fam. XXI 10, 16-21) Petrarca racconta a Gugliemo da Pastrengo della ferita "ciceroniana» che gli aveva procurato alla gamba l'urto frequente con il pesante codice di Cicerone che teneva appoggiato allo stipite della porta: ebbene, a curarla egli è ricorso anche al digiuno, quale alleato della natura («adiuta per abstinentiam natura»), sottolineando ancora una volta che la ferita è qualcosa di altrettanto «innaturale» che il mangiar troppo, e che la frugalità per contro contribuisce a risarcire la natura, nel caso quella del suo corpo, della violenza che abbia subìto. ${ }^{51}$

\section{IV}

Una volta constatato (con relativa facilità, occorre dire) che Petrarca pone ripetutamente la coppia: vita solitaria — frugalità alla base di una vita serena, caratterizzata da salute fisica e mentale e dedita tanto ai piaceri quanto ai doveri dello spirito, altre considerazioni si impongono. Intanto, sembra abbastanza evidente che ci troviamo dinanzi a una riuscita e in gran parte originale mescolanza di istanze classiche e cristiane, dove l'originalità non sta nelle singole componenti, tutte ben note alla tradizione, ma piuttosto nel gioco sottile del loro reciproco adattamento. Petrarca rende omaggio (Fam. XXII 14) alla frugalità militare che va annoverata tra le qualità maggiori degli eserciti romani e dei suoi generali (topica la frugalità di Giulio Cesare e di Augusto), e fa corpo unico con il loro senso della disciplina e la loro statura morale: ricordiamo, al proposito, che proprio in nome di una concezione larga di tale frugalità Petrarca aderisce senza riserve alla tesi della superiorità degli eserciti romani su quella banda di intemperanti ubbriaconi che costituivano l'esercito di Alessandro Magno, secondo quanto già sosteneva Livio nel famoso excursus del l. IX delle sue Storie, né manca per contro di elogiare, nel De viris, la rigidissima frugalità di Annibale. Ma ciò non è, appunto, che un ingrediente, anche se molto importante all'interno della sua visione complessiva, che conosce interessanti sfumature. È lo stoicismo di Seneca, infatti, che sta alla base del concetto di una natura regolatrice e ordinatrice alla quale il saggio deve conformarsi, e che condanna come innaturale ogni eccesso non sorvegliato da una superiore istanza equilibratrice della quale è appunto la natura a custodire la chiave. Ma è poi la fine polemica di costume condotta da Orazio, alla quale aggiungono punte più forti Giovenale e Persio, che colora di sé l'ideale di una mediocritas che diventa misura di vita e modello estetico. E tutto ciò è però continuamente riportato entro la cornice della morale cristiana che sulla scorta delle condan-

50. Opere latine, p. 594 e 746-748.

51. Per questa insistenza «salutista» vedi CASAGRANDE-VECCHIO, op. cit., p. 138-139, che danno risalto alla posizione in merito di Egidio Romano, nel terzo dei suoi Sermones de tribus vitiis mundi (AEGIDII ROMANI Opera omnia. I, Repertorio dei Sermoni, a cura di Concetta Luna, Firenze: Olschki, 1990, p. 375-378). 
ne bibliche contro chi del proprio ventre fa Dio (Ad Philipp. 3, 19, e Ad Rom. 16, 18; Ecli. 37, 30-31, ecc.) aveva dedicato molte pagine al peccato di gola, annoverato tra i capitali. ${ }^{52}$ Anche in questo caso i testi che fanno al caso sono moltissimi, ma converrà restare ai fondamentali tra i quali stanno certamente il più volte citato, da Petrarca, Adversus Iovinianum di Gerolamo, e il De Elia et ieiuno di Ambrogio ( $P L$, rispettivamente XXIII e XIV), e soprattutto i Moralia in Job di Gregorio Magno (in particolare 30, 18).

Per intendere Gregorio è tuttavia opportuno partire da Agostino: più precisamente, dalle perplessità di Agostino. Il quale aveva scritto di non temere tanto l'impurità del cibo, ma quella della cupidigia di cibo, dal momento che egli coglieva la inquietante presenza di un piacere che s'accompagna alla necessità di nutrirsi: un piacere dal quale occorre non farsi catturare, ${ }^{53} \mathrm{ma}$ che è ineliminabile dall'atto stesso con il quale si soddisfa alla necessità, e complica dunque ogni discorso sul peccato di gola, dal momento che è difficile distinguere se sia la necessità fisica che vuol essere soddisfatta legittimando il piacere che ne deriva, o sia piuttosto il piacere che subdolamente si maschera come necessità ${ }^{54}$. Al punto da fargli concludere di non avere ancora idee chiare sulla questione ("consilium mihi de hac re nondum stat»). Così, da un lato anche Gregorio risolve la questione psicologizzando il peccato e riconducendolo a un colpevole eccesso di desiderio che può volgersi benissimo anche a cibi poveri e semplici (Adamo si è perduto per una mela ed Esaù ha ceduto la primogenitura per un piatto di lenticchie, e insomma «spesso mangiamo senza colpa cibi più ricchi, e ne gustiamo invece di umili commettendo peccato di coscienza»), e dall'altro s'arrende impotente dinanzi all'osservazione che già Agostino aveva fatto: "Nel momento stesso in cui si soddisfa la necessità naturale, il piacere di mangiare si mescola a tale esigenza: che cosa essa chieda e che cosa reclami il piacere non si riesce a distinguere». ${ }^{55} \mathrm{La}$ sua soluzione è in qualche modo doppia: da una parte il peccato di gola, fermamente condannato e associato da stretta parentela con la dissolutio spiritus e la immunditia luxuriae (Hom. in Hiezechihelem I 12), può essere combattuto cercando di non superare mai il limite della necessità naturale; dall'altro occorre sapere che in ogni caso, se si vuole mantenere il corpo in grado di fare buone azioni e di pregare, si rischia una situazione paradossale, dal momento che, diremmo, il cibo nutre egualmente sia i vizi che le virtù, e l'astinenza indebolisce egualmente quelli e queste:

52. Al peccato di gola è dedicata la voce Gourmandise et gourmandise spirituelle di William YEOMANS e André DERVILLE nel Dictionnaire de spiritualité, VI p. 612-626, con utile rassegna di testi patristici.

53. Conf. X 31, 43: «suavis est mihi necessitas, et adversus istam suavitatem pugno ne capiar».

54. Ibid. 44: "quanto basta alla salute è poco per il piacere, ed è spesso difficile stabilire se siano le necessità del corpo a invocare aiuto, o se non sia invece una sorta di autoinganno generato dal desiderio a darsi da fare per la propria soddisfazione» [saepe incertum fit utrum adhuc necessaria corpori cura subsidium petat an voluntaria cupiditatis fallacia ministerium suppetat].

55. «Unde et lautiores cibos plerumque sine culpa sumimus, et abiectiores non sine reatu conscientiae degustamus; "Eo enim tempore quo necessitati debitum solvitur, quia per esum voluptas necessitati miscetur, quid necessitas petat et quid [...] voluptas suppetat, ignoratur». 
spesso mentre inseguiamo il nemico, uccidiamo anche il concittadino che amiamo, e spesso mentre risparmiamo il concittadino nutriamo il nemico che ci combatte. Infatti i vizi crescono con gli stessi alimenti di cui si nutrono le virtù, sì che mentre si nutre la virtù i vizi spesso aumentano le loro forze. All'opposto, quando una illimitata astinenza indebolisce la forza dei vizi, anche la virtù ansima in difficoltà. ${ }^{56}$

Ed è per uscire con qualche indicazione più precisa dal dilemma che Gregorio propone una casistica che specifica in cinque punti le modalità del peccato di gola, che si ha quando ci si getta a mangiare prima del tempo e quando si mangia con eccessivo lusso; con troppa ricercatezza di gusto; in quantità esagerata; con infinita bramosia ("aestu immensi desiderii») ${ }^{57}$, così come in altri cinque punti egli ne compendia gli effetti: sciocca allegria; scurrilità; multiloquio; impurità; ottusità mentale (Mor. In Job. 31, 45).

Mi sono fermato alquanto sulle pagine di Gregorio perché sono quelle che hanno fatto testo per secoli: ad esse, per esempio, ricorre ancora Tommaso, nella sua ampia Quaest. XIV de gula, art. 3, delle Quaestiones disputatae de malo, e perché danno l'idea, mi pare, dello sfondo che sta dietro Petrarca e dal quale egli si stacca, pur muovendo proprio da quell'attenzione alla inevitabile parte che il gusto rivendica a sé secondo Agostino. Il quale è ovviamente fermo sulla necessità di non essere schiavi del proprio corpo, ma è anche disposto ad ammettere che in questo campo un moderato eccesso, se l'ossimoro è accettabile, sembra pur esso far parte della natura dell'uomo. ${ }^{58}$ E proprio perché sembra toccare da vicino Petrarca, il punto merita una breve sosta, che arrichisce il quadro sin qui sommariamente tracciato.

A un certo punto del De vita solitaria, nel libro secondo, ${ }^{59}$ Petrarca si sofferma sul De moribus brachmanorum (PL XVII, col. 1167 s.), libera versione del testo greco di Palladio ma attribuito allora ad Ambrogio. Petrarca mette le cose a posto per quello che riguarda l'autore, giudicando sulla base di solidi indizi che il libro sia appunto di Palladio, e riferisce con notevole cura quello che il libro dice dei costumi dei bramani, mescolando approvazione e condanna. L'approvazione va al loro disprezzo del mondo e dei suoi beni, alla solitudine,

56. «Saepe vero dum in illo hostem insequimur, etiam civem quem diligimus trucidamus; et saepe dum quasi concivis parcimus, ad proelium hostem nutrimus. Eisdem namque alimentis vitia superbiunt quibus nutritae virtutes vivunt. Et cum virtus alitur, plerumque vires vitiis augentur. Cum vero immensa continentia vitiorum vires extenuat, etiam virtus deficiens anhelat».

57. Raterio veronese semplificherà in tre punti, Sermo II de Quadragesima, p. 68, 118: «il primo peccato di gola sta nel mangiare prima del tempo; il secondo mangiare a tempo ma troppo; il terzo, andare in cerca di cibi troppo ricchi e squisiti" [primum eius [della gola] vitium est ante horam manducare; secundum in hora plurimum manducare; tertium lautiores et delitiosos cibos exquirere]. Vedi avanti, nota 72, per l'analogo elenco, in sei punti, di Egidio Romano.

58. Conf. X 31, 47: "Chi è, Signore, che non si lascia trascinare un poco oltre i limiti del necessario?» ["Et quis est, Domine, qui non rapiatur aliquantum extra metas necessitatis?»]. Ma si veda tutto il paragrafo.

59. Opere latine, p. 486-494. 
alla libertà, al silenzio, alla meditazione ... La condanna colpisce invece alcune inaccettabili estremizzazioni: la pratica del suicidio, l'arroganza del credersi perfetti, la nudità che offende il pudore, e:

Non mi piace la loro animalesca indifferenza per il sonno e il cibo: non accada che mentre rifuggiamo dagli estremi di una vita troppo raffinata finiamo per cadere nel contrario. E mi piace invece, qui come in altri casi, la via di mezzo di Cicerone, che dice: «Occorre avere una cura di sé né oltraggiosa né troppo raffinata, ma sufficiente a evitare una sciatteria rozza e disumana» [De off. I 130]. È il criterio medesimo che va impiegato nell'abbigliamento, rispetto al quale la cosa migliore, come come nella maggior parte delle cose, è la giusta misura. Questo è il modo di vita che mi piace. Il sonno sia breve, leggeri i cibi, naturali le bevande, modesto il vestire, e insomma ci sia differenza tra l'abbigliamento, il letto, la tavola dell'uomo, e quelli di un branco di animali [...] Trascorrere l'intera vita a cielo aperto giudico sia cosa da orsi, e non da uomini. ${ }^{60}$

Ora, riesce affatto inevitabile riportare queste affermazioni di principio, implicitamente ribadite poco avanti parlando dei modi di vita degli abitanti delle Isole Fortunate (le Canarie), sia a Seneca, per es. Ad Luc. 5, 4-5, ove costui dichiara che è contro natura torturare il proprio corpo, vivere nel sudiciume e cibarsi in modo disgustoso: una vita "filosofica», infatti, comporta la frugalità, non l'afflizione («Frugalitatem exigit philosophia, non poenam»), sia alla limitazione moderatrice con la quale Agostino, nel Secretum, nel passo sopra citato, riferisce le parole di Seneca circa il pane e l'acqua ai quali la dieta umana potrebbe e dovrebbe limitarsi, e dichiara il suo fastidio per ogni estremizzazione, sospetta per di più di essere meramente retorica. Quel magnus opinator che Petrarca dice di essere rifugge evidentemente da ogni fanatismo, specie se puramente verbale, e di questo suo atteggiamento volto contro gli eccessi rigoristici della morale stoica e fondato essenzialmente su Cicerone seguace dello stoicismo «moderato» di Posidonio e Panezio, danno testimonianza, per esempio, le Fam. XVI 6, 14-15, e XXIII 12, 6 s. ${ }^{61}$ Ma sembra ragionevole pensare, nel caso, che sullo sfondo agisca quel medesimo scrupolo di realistica saggezza al quale Agostino già dava voce ("Chi è, Signore, che non si lascia trascinare un poco oltre i limiti del necessario?»), e che dava da pensare anche a Gregorio Magno, giustamente perplesso quando non riusciva a dividere con un taglio troppo netto le ragioni della natura da quelle del piacere. Ma l'esito di questo discorso va oltre e ci porta a un altro dei suoi ingredienti, che finisce per mar-

60. «Non placet incuriositas belluina somni cibique, ne dum extremum curiose vite fugimus in contrarium relabamur; placet hac in re, ut in multis, ciceroniana temperies: "Adhibendainquit- est munditia non odiosa neque exquisita nimis, tantum que fugiat agrestem et inhumanam negligentiam”. Eadem ratio est habenda vestitus, in quo sicut in plerisque rebus mediocritas optima est. Hic, inquam, michi vite modus placet. Sit somnus brevis, cibus levis, potus facilis, humilis toga, modo aliquid inter habitum ac thorum mensamque hominis et pecudis intersit [...] Totum vero sub divo tempus agere, non tam hominum quam ursorum iudico».

61. Vedi anche Secretum, ed. cit., I, nota 18, e II, note 99 e 142, p. 295, 333 e 338. 
care l'originalità e l'importanza della posizione di Petrarca: originalità che non sta tanto, ripeto, nelle singole affermazioni, che potremmo spesso dare addirittura per banali, ma piuttosto, com'è in genere tipico di lui, nel modo libero e intelligente con il quale sono articolate all'interno di un più ampio orizzonte culturale.

Già ho anticipato l'osservazione che in Petrarca l'aspetto concreto della pratica alimentare gode di autonomia ed attenzione particolare, ponendosi come un momento della vita in grado di definire i propri fini e di definire un sistema razionale di regole che valgono per sé, in relativa indipendenza dal pur immanente aspetto morale. Ora, ci riesce più facile precisare che è proprio in nome di uno «specifico naturale» e della forza con la quale esso genera un insieme di regole, e in definitiva una sua specifica morale, che l'insieme delle scelte petrarchesche non riposa affatto su esigenze di tipo ascetico: non risponde, infatti, a una logica di rinuncia, così come fuoriesce dal quadro tradizionale rigidamente ancorato alla nozione di peccato. Al contrario, esse si pongono come scelte positive: come le scelte giuste contro le scelte sbagliate, allo stesso identico modo di quella lunga serie di opposizioni strutturali: barbarie vs civiltà; vita cittadina $v s$ vita solitaria; confusione vs silenzio; esteriorità $v s$ interiorità; lusso $v s$ misura; sporcizia $v s$ pulizia, e via elencando, sino al mangiar troppo vs il mangiar poco. Tout se tient, abbiamo detto: solo così infatti si realizza un compiuto ideale di vita fondato sulla tranquillità dello spirito, la bellezza della natura, la purità dell'aria, il silenzio, la sana e risanante freschezza dell'acqua... E come se nel gusto e nella misura naturali si attui una redenzione dal quotidiano, nella quale lo spirito si fa misura concreta e visibile della realtà, e la materia infine dominata acquista la leggerezza e la trasparenza che rendono sopportabile la vita.

Sentiamo ancora una volta Petrarca stesso, che torna a ribattere sullo stesso chiodo nella già segnalata Fam. XIII 4, 24, là dove esalta quelli che, come già facevano gli antenati,

considerano il cuoco come il più vile degli schiavi; che hanno il coraggio di domare con lunghi digiuni quei servi insolenti e pigri che sono la gola e il ventre; che si sono abituati a un pane duro e grossolano composto d'ogni genere di cereali; che non schifano di bere acqua pura di sorgente e di mangiare cibi rustici, doni spontanei della madre terra, né disprezzano le erbe e le bacche degli alberi e i dolci frutti; che riescono a vivere senza uccidere animali; che chiedono aiuto per lo stomaco stanco e malato non all'abbondanza di cibi raffinati ma alla frugalità, all'appetito e all'esercizio fisico; infine, che non sono schiavi del corpo ma sanno dettargli delle regole e farle rispettare. ${ }^{62}$

62. «vilissimum mancipium coquum putent; qui gulam ac ventrem ceu contumaces et inertes servos longo ieiunio domare audeant, durum et inelaboratum atque ex omni fruge compositum panem sibi familiarem fecerint; neque haustum puri gurgitis horreant neque victum agrestem, terre matris munera sponte nascentia, herbas atque arborum baccas et dulcia poma fastidiant, possintque sine animantium interitu suam aliquando vitam agere; qui ad auxilium fessi ac languentis stomaci non copiam mollesque delitias sed abstinentiam et famem et exercitium implorent; postremo qui corpori non servire sed leges dare atque imperare didicerint». 
É precisamente e solo a questo punto che può entrare in scena l'aspetto propriamente dietetico, o meglio, che si può parlare di una dietetica petrarchesca quale parte importante dello stile della vita. E ciò ci porta alle Seniles, che finiscono di dare ai motivi tratteggiati sin qui tutto il loro spessore, in pagine nelle quali la retorica di Petrarca diventa davvero voce e sostanza del suo umanesimo. Anche in questo caso lo spettro delle possibili citazioni è molto ampio, ma vorrei soffermarmi brevemente almeno sulle cinque lettere che già sopra ho elencato: sono, ripeto, la V 3, al Boccaccio (1365); le due, XII 1 e 2, a Giovanni Dondi (1370); la XV 3 a Lombardo della Seta (1373); la XVI 3 al medico Francesco Casini da Siena (1374). Lettere tarde e tardissime, dunque, scritte, meno quella al Boccaccio, all'ombra delle proprie incerte condizioni di salute: lettere nelle quali l'attenzione in parte nuova riservata al declino del corpo colora in maniera persino commovente quel grande testamento spirituale che le Seniles nel loro complesso sono.

Non ho qui lo spazio per raccogliere quanto esse offrono, in generale e in particolare, e mi limiterò dunque a puntualizzare poche cose che possano arricchire, sia pure provvisoriamente, la linea di discorso sin qui seguita. Il che comporta, per esempio, che terrò fuori il motivo più evidente e noto, costituito dalla forte polemica contro i medici (così come ho tenuto fuori il pur pertinente capitolo contro il vino, che ha un risalto tutto speciale - lo si sa beneall'interno della polemica anticuriale), anche se, per cominciare, la lettera a Boccaccio va sotto il titolo De audacia et pomposo medicorum habitu, ed effettivamente appare come una sorta di completamento o «coda» delle Invective contra medicum. Estraiamone qualche elemento. Petrarca inveisce contro quei medici che proibiscono di mangiare frutta, legumi ed ortaggi, e contro coloro che proibiscono di bere acqua: per quanto sta a lui, ne ha sempre bevuto moltissima, fredda, anche d'inverno, e sente che è assolutamente indispensabile alla sua salute e addirittura alla sua sopravvivenza. Quanto alla frutta, poi, ne è sempre stato ghiottissimo e, specialmente in gioventù, non sapeva rinunciare a quella non perfettamente matura e ancora un po' acida. Insiste in maniera particolare sul fatto che il paziente è il miglior medico di se stesso, perché ha lunga esperienza di sé e di ciò che la sua complessione richiede, e tra altre cose ricorda che l'imperatore Aureliano non voleva medici attorno a sé, e si curava con il digiuno. Ora, per quanto possano complicarsi e ramificarsi le affermazioni contenute nelle lettere successive, questi sono i punti fermi continuamente, ostinatamente ribaditi, che caratterizzano la sua posizione: difesa ad oltranza della pratica del digiuno, e difesa dell'acqua fredda, della frutta e degli ortaggi quali alimenti-base, solo moderatamente integrabili, di tanto in tanto, con della carne (il formaggio, che pure compare all'interno di qualche esempio - quello di vacca nel caso di Augusto, e il «caseus sardus» nell'apologo di Fam. III 13, 2 - non sembra invece godere di molta considerazione). ${ }^{63}$ Circa la carne, 
per la verità, abbiamo colto la traccia del rifiuto "politico" implicito nell'esaltazione e nell'offerta della frutta e dei pesciolini di Valchiusa, ma anche del rifiuto che parte dal disgusto del tutto evidente nelle pagine citate del De vita solitaria e si fa via via più radicale, e si carica di risonanze più complesse. Petrarca riconosce di averne sempre mangiata, seppure, appunto, con moderazione e ad intervalli di tempo, ma nella Fam. XIII 4, citata poco sopra, elogia chi non ha bisogno, per vivere, di uccidere gli animali, e questa posizione è infine ripetuta con un' enfasi e una radicalità affatto particolari nella Senile XV 3 a Lombardo della Seta, ove il mangiare carne è considerato quasi atto di manifesta empietà, sulla scorta di una citazione di Virgilio, Georg. II 536-538. ${ }^{64}$ Ahimè, ne mangio anch'io, dice Petrarca, ma

oserei pur io definirlo, con Virgilio, il cibo degli empi. Egli infatti, descrivendo l'età dell'oro prima di Giove, principio di tante disgrazie e di tanti errori, scrive che avanti il regno del re Ditteo, prima che le empie genti si cibassero di giovenchi sgozzati, l'aureo Saturno conduceva in terra questa vita: cioè una vita parca e modesta che s'accontentava dei frutti della terra e s'asteneva dall'uccidere gli animali, in ispecie terrestri. Ma tutto cominciò a mutare sotto Giove, e poco a poco si è arrivati al punto che non si risparmiano non solo i giovenchi ma nessun altro animale, sì che persino i più schifosi e i più orrendi e addirittura i velenosi vengono messi alla prova della tavola, e l'uomo che altro non è che un animale terrestre ha finito per convincersi che non può vivere se non a prezzo della morte delle altre creature della terra. ${ }^{65}$

Il rifiuto della carne, luporum cibus in Sen. XII 1, 22, è interessante perché le ragioni, in Petrarca, hanno poco di religioso, ${ }^{66}$ e rinviano piuttosto a una morale che è «naturale» — una morale da età dell'oro, appunto, quando nessuno

21, 1-2: «Era molto goloso di frutta e di verdura e assai parco negli altri cibi, tanto che mangiava in continuazione qualche frutto non ancora del tutto maturo. Era avido di bevande fredde e durante l'estate non beveva altro e in gran quantità" [Pomorum et olerum avidissimus fuit, in reliquo ciborum genere parcissimus, ut semper pomorum aliquid recentium devoraret. Figidarum percupidus nec facile per aestatem nisi frigidas et quam plurimas bibit]. Per la petrarchesca passione per la frutta acerba, vedi Sen. XII 2, in Opera omnia, p. 10041005.

64. Ma vedi pure Cicerone, De nat. deorum II 159, con cit. di Arat. 129-132; Varrone, De re rustica II 5, 3-4.

65. «auderem et ego cum Virgilio impiorum cibum appellare. Sic enim ait, illud ante Iovem multorum malorum principem atque errorum tempus aureum describens, "ante etiam sceptrum Dyctei regis, et ante / impia quam cesis gens est epulata iuvencis / aureus hanc vitam in terris Saturnus agebat". Vitam scilicet parcam et modestam et terre frugibus contenta et animalium cedibus abstinentem, precipue terrestrium. Mutata res sub Iove primum, eoque sensim deducta est ut non solum iam iuvencis, sed nulli parcatur animantium, ita ut iam feda etiam et horrenda, insuper et venenosa, tententur, persuasumque est homini, qui et ipse terrestre est animal, quod sine terrestrium morte vix viveret».

66. Opera omnia, p. 1033. Di là dall'autorizzazione biblica, la carne resta infatti un cibo in linea di principio interdetto ai religiosi: vedi per ciò (e per molto altro, date le ampie implicazioni dell'argomento) MONTANARI, op. cit., in particolare il cap. IV, Mangiare gli animali, p. 35-62, e il V, Diete monastiche, p. 63-104. 
uccideva per mangiare- e insieme culturale e fortemente elitaria. Si ricordi, infatti, che la Chiesa aveva assai presto abolito ogni vecchio tabù che era nel Levitico e nel Deuteronomio, e che per essa tutto, assolutamente tutto è mangiabile: negli Atti degli Apostoli, 10, 10-15, è Dio stesso che fa apparire agli occhi di Pietro affamato una sorta di contenitore nel quale erano "omnia quadrupedia et serpentia terrae et volatilia caeli» e gli dice: "occide et manduca», mettendo a tacere le perplessità di Pietro che sino a quel momento non ha mai mangiato animali impuri o immondi (ma niente è impuro in sé, come dice Paolo, Ad Rom. 14, 14, mentre in I Cor. 10-25 precisa che tutto ciò che arriva al mercato è commestibile e non deve suscitare problemi di coscienza). ${ }^{67}$ In ogni caso questo della carne non è un elemento centrale, anche se l'indubbio radicalismo estetico di cui Petrarca l'investe è coerente con la difesa del digiuno e l'uso di mangiare frutta e ortaggi e di bere acqua fredda, nel momento in cui le sue condizioni di salute, caratterizzate a quanto egli ci fa sapere da improvvise e violente febbri, si aggravano e i medici gli si fanno attorno con le loro prescrizioni. Siamo con ciò alle due lunghe Seniles XII 1 e 2, scritte «in stato di grave e complicata malattia, e forse vicino alla morte» (Sen. XII 1), ${ }^{68}$ e indirizzate con tono di affettuosa ma ferma polemica all'amico medico e scienziato Giovanni Dondi, dedicate in maniera specifica all'argomento. Anche in questo caso mi limiterò a mettere in rilievo i pochi punti che corrispondono a quanto detto sin qui, avvertendo tuttavia che si tratta di testi che potrebbero reggere, da soli, tutto il peso dell'argomentazione, tanto bene Petrarca riassume in essi la sua posizione e la inquadra nella sua complessiva esperienza di vita. ${ }^{69}$ Il Dondi si preoccupa molto della salute del suo illustre amico, e cerca di fargli mutare le sue abitudini alimentari: in particolare, a quanto si capisce, egli vorrebbe che Petrarca, che non sta affatto bene, riducesse i digiuni che l'indebolivano troppo; eviti la verdura cruda, la carne e i pesci salati (in questo caso Petrarca obbedisce, né la cosa gli costa) e la frutta, e non beva acqua fredda (è molto probabile infatti che sia proprio lui il medico del quale Petrarca parla all'altro medico, Francesco Casini, nella Sen. XVI 3: «quando un medico mi entra in casa, so già cosa mi dirà: mangia polli novelli e bevi acqua bollita intiepidita»). ${ }^{70}$ Insomma:

per te e per tutti i medici che ti fanno coro bere acqua è l'unica o la principale ragione del mio male. Viva dunque l'ubbriachezza! Altri aggiungono il man-

67. «Omne quod in macello venit manducate nihil interrogantes propter conscientiam», ecc. Sul punto, vedi anche AGOstino, Conf. X 31, 46.

68. «in morbo gravi ac multiplici et nescio quam vicinus morti». La cito da F. P., Epistole autografe. Introduzione trascrizione e riproduzione a cura di Armando Petrucci, Padova: Antenore, 1968 , p. 40-51.

69. Aggiungo che la relazione prevedeva a questo punto un approfondimento sugli aspetti propriamente medico-dietetici del discorso, ma vi ho rinunciato perché avrebbe troppo gravato sul resto. Mi riprometto tuttavia di ricavarne al più presto un breve saggio a sé, anche perché mi sono trovato dinanzi a qualche elemento che interessa da vicino Petrarca.

70. Opera omnia, p. 1053-1054: «antequam meum limen intret medicus, scio ex parte quid consilii afferat: Ede pullos vix dum natos, bibe aquam coctam tepentemque». 
giare frutta, l'evitare la carne, il digiuno [...] Un altro dei consigli non tanto tuoi quanto di tutti gli altri medici (ma immagino che tu, sapendo di avere a che fare con un bastiancontrario come me, ti tieni coperto: nelle prescrizioni degli altri io tuttavia riconosco anche la tua, anche se tu la emetti con più garbo e quasi di passaggio) è che io mi astenga dalla frutta e da qualsiasi genere di frutto arboreo quasi fosse aconito o cicuta. ${ }^{71}$

Nulla di strano, però, in queste proibizioni, che non fanno che ripetere quanto universalmente consigliava la pratica medica del tempo, basata sul Canone di Avicenna, citato passo passo in un testo che potremmo ben assumere come rappresentativo di moltissimi altri, e che segnò il punto di svolta nella storia della dietetica occidentale: il commento alle regole del Regimen sanitatis salernitano di Arnaldo da Villanova. ${ }^{72}$ Qui per la verità troviamo varie cose, nel Regimen prima di tutto e poi nell'ampio commento, con le quali Petrarca si trova in sintonia: l'elogio della frugalità; l'idea, che in Petrarca è centrale nella sua polemica col Dondi, che per quanto è possibile è meglio non cambiare le proprie abitudini, specie se si è vecchi (c. 131v: «Mutare consuetudinem nocivum est et molestum, et precipue senibus»); la scelta, se si mangia una sola volta al giorno, di farlo verso sera (si ricordi il citato Gerolamo: sia il pranzo "vespertino"); non mangiare se non sia stato completamente digerito il pasto precedente, perché non c'è niente di peggio per il corpo che accumulare nuovo cibo sull'altro non ancora digerito (c. 132: «nihil est deterius in humano corpore quod intromittere cibum super alium qui non est digestus sed digeri incipit»): e l'occupatus, abbiamo visto, è precisamente descritto come la prova vivente (meglio, morente!) di ciò che accade a chi non rispetta questo principio. Il quale principio ha alcune importanti conseguenze. È infatti difficile rispet-

71. Sen. XII 1, 22 e 33: «Audio iam hinc te [il Dondi] et concinentes undique medicos aque potum unam esse vel precipuam mali causam. O felix ebrietas! Addent alii pomorum esum, fuga carnium, ieiunium [...]Aliud ex consiliis non tam tuis quam medicorum omnium (tu enim sciens, credo, cum litigioso homine tibi rem esse, cautius agis, sed ex aliorum sententiis tuam metior, quam tu quoque lentius vibras et in transitu), est autem ut pomis et omni genere fructuum arboreum quasi aconito abstineam aut cicuta».

72. Lo cito dall'edizione dell'Opera omnia del famoso medico, Lione: Fradin, 1505, cc. 130v150. Sull'opera, vedi Juan Cruz CruZ, Dietética medieval. Apendice con la version castellana del Regimen de salud de Arnaldo de Vilanova, Huesca: La Val de Onsera, 1997, p. 217-224; Melitta Weiss Adamson, Medieval Dietetics. Food and Drink in "Regimen Sanitatis» Literature from 800 to 1400, Frankfurt-am-Main: Lang, 1995, p. 110-117. Sul rapporto medicinadietetica nel medioevo esiste ormai una bibliografia vasta e specializzata. Qui, mi limito a rinviare a due ricchi saggi d'insieme: il primo, della medesima studiosa Melitta WEISS, «The role of medieval Physicians in the diffusion of Culinary recipes and cooking practices», in Carole LAMBERT (a cura di) Du manuscrit à la table. Essais sur la cuisine au Moyen-Âge et répertoire des manuscits médiévaux contenant des recettes culinaires, Montréal-Paris: Les Presses de l'Univ. De Montréal-Champion/Slatkine, 1992, p. 69-80, che mette ordine nella serie dei testi di medicina; il secondo, recente, di Ilaria ZAMUNER, «Il ms. Barb. Lat. 311 e la trasmissione dei regimina sanitatis (XIII-XV sec.)", Cultura neolatina, LXIV, 2004, p. $207-$ 250: si tratta di una fondamentale indagine (anche per la sistematica e preziosa bibliografia) che dà un quadro articolato e completo della tradizione dei testi di dietetica, la cui fortuna è ricondotta all'ambiente pontificio e alla corte di Federico II. 
tarlo se i pranzi sono troppo lunghi e se tale esagerata lunghezza è occupata a divorare cibi di diverso genere, che hanno tempi di digestione diversi:

Si deve inoltre sapere che durante il medesimo pasto è cosa pessima mescolare molti cibi diversi, come carne, pesci, polli e maiale, e prolungare il tempo del pasto, poiché quando il primo cibo comincia a essere digerito vi si sovrappone l'ultimo sì che nel processo della digestione si crea un contrasto tra le diverse parti di cibo, ${ }^{73}$

sì che anche sotto questo aspetto le colorite descrizioni del De vita solitaria, soprattutto, riposano sulla base consolidata del normale sapere medico. Le differenze tuttavia emergono quando si entra nello specifico e si osserva che, dal punto di vista medico, c'è in genere molta diffidenza se non proprio ostilità verso la frutta e gli ortaggi, decisamente interdetti agli infermi. Il motivo di fondo è il medesimo: la loro natura di elementi freddi e «acquosi» raffredda quella specie di pentola naturale che è lo stomaco, e rallenta e corrompe la digestione. Dice il Regimen che pesche, mele e pere sono nocive agli ammalati per la loro natura «melanconica» («Persica, poma, pira [...] hec melanconica sunt infirmis inimica»), e Arnaldo conferma, citando Avicenna, che la frutta tende a fermentare nello stomaco corrompendo il processo digestivo ( $\mathrm{E}$ t omnes fructus nocent febricitanti cum ebullitione sua et corruptione in stomacho»), ed è dunque particolarmente interdetta a chi ha la febbre (ma, occorre dire, nella trattatistica medica paiono fare eccezione i fichi, provvisti di quella capacità nutritiva che all'altra frutta fa difetto). ${ }^{74}$ Tutto ciò vale in maniera speciale per l'acqua. Infatti:

73. c. 132: «Sciendum est insuper in una et eadem refectione pessimum est simul multos et diversos coniungere cibos, ut carnes et pisces vel pullos et porcum, et postea prolongare tempus in comedendo, quia primus cibus iam digeri incipit cum advenit ultimus, et sic partes cibi dissimiles fiunt in digerendo». Il punto è sottolineato, tra molti, anche da Egidio Romano, là dove elenca le sei maniere con le quali si pecca nel mangiare: «La quarta maniera si è, che l'uomo die mangiare ad ora e a tempo ordinatamente, ché mangiare innanzi l'ora pare una ghiottezza; e somigliantemente è molto nocevole al corpo, perciò che mèttare vianda sopra vianda, anzi che quella sia cotta, s'impedisce la digestione naturale, unde ne nascono molte infermità; donde quelli che vuole mantenere la sanità del suo corpo non die mangiare, sed elli non à ismaltito quello ch'elli à mangiato di prima» (gli altri peccati sono nel mangiare in fretta senza masticare; nel mangiare troppo; nel mangiare laidamente, insozzandosi; nel volere cibi troppo fini rispetto alla propria condizione; nel volere lusso di apparati: vedi sopra, n. 56). Cito da Del reggimento de principi di Egidio Romano, volgarizzamento trascritto nel MCCLXXXVIII, pubblicato per cura di Francesco Corazzini, Firenze: Le Monnier, 1858, p. 173-174 (II, ii, 11). E la stesa cosa è già nella diffusissima Epistula Alexandri de dieta servanda: «bada a non mangiare sin che tu non sia assolutamente sicuro di avere lo stomaco vuoto, ben ripulito dal pasto precedente» [Et cave ne iterum comedas donec certissime cognoscas stomacum esse vacuum, id est purgatum a primo cibo]. Cito dal testo fornito da Lucilla SPETIA, "Un nuovo frammento dell' "Epistola Aristotelis ad Alexandrum”", Studi Medievali, 3o s., XXXV, 1, 1944, p. 405-434 (il testo, p. 426-432: la cit., p. 430).

74. Si veda ancora, a mero titolo d'esempio, il volgarizzamento del Régime du corps di Aldobradino da Siena (circa 1310) di Zucchero Bencivenni: «si de l'uomo astenere di tutti frutti, perciò che ciò è la cosa che più fano febri ingienerare; che, sì come disse Galieno, che 
L'azione dello stomaco sul cibo ingerito si esplica contemporaneamente nel consumarlo e nel cuocerlo: nello stomaco avviene esattamente quello che capita quando si versa dell'acqua in gran quantità in una pentola per ritardare la cottura del cibo. ${ }^{75}$

Per questo, l'acqua è tutt'al più ammessa in piccole dosi prima del pasto, ma proibita sul cibo: nello stomaco, con i cibi combina molto meglio il vino! ${ }^{76}$ Ma infine, teste ancora Avicenna, l'acqua è nociva in vari altri casi: durante il digiuno; dopo un faticoso esercizio fisico e dopo il coito; dopo il bagno; per calmare quella falsa sete notturna che tormenta chi ha mangiato e bevuto troppo, e insomma «in genere fa male alle persone sane bere molta acqua fredda, perché spegne il calore naturale, danneggia il cuore, scaccia l'appetito dello stomaco e nuoce alle parti nervose». ${ }^{7}$

L'argomento non è naturalmente riducibile a questi pochi spunti, che per il momento sono però sufficienti per tornare a Petrarca e valutare meglio quanto egli dice. La difesa delle sue abitudini dinanzi alle richieste per altro moderate e ragionevoli del Dondi è ferma, e probabilmente inquinata da una vena di senile ostinazione e petulanza proprio là dove è pur obbligato a riconoscere la sostanza dei consigli che gli venivano dati. Eccolo infatti, nella XII 2 allo stesso Dondi, ribattere d'essere in grado di conoscere da solo lo stato della propria salute, e vantarsi di godere ancora di buona digestione:

quelli non ebe unque febre che no mangiò unque frutti. E si conviene ghuardare di bere aqua freda ...». Cito da Rossella BALDINI, "Zucchero Bencivenni, "La santà del corpo". Volgarizzamento del "Régime du corps" di Aldobrandino da Siena (a. 1310) nella copia di Lapo di Neri Corsini (Laur. Pl. LXXIII 47)», Studi di lessicografia italiana, n. 15, 1955, p. 21-300: la cit., p. 109.

75. c.137v: «Actio namque stomachi super sumptum cibum fit conterendo et coquendo simul, unde sicut infunditur simul et in magna quantitate aqua in olla propter quod tardatur cibi decoctio in eadem: sic etiam contigit idem ipsi stomacho".

76. c. 137v: «il vino giova di più alla digestione in ragione del calore che produce, mentre l'acqua la impedisce a causa della sua freddezza. E dunque evidente che il vino è superiore all'acqua nel mescolarsi ai cibi» [vinum magis iuvat ratione virtualis caliditatis, et aqua impedit ratione frigiditatis. Et sic patet quod vinum prevalet aqua in ciborum permixtione].

77. c. 138v: "generaliter malum est sanis bibere multam aquam frigidam quod extinguit calorem innatum et pectus offendit et stomachi appetitum deijcit et membris omnibus nervosis obest». E la citata Epistula Alexandri, p. 429: «Ugualmente trattieniti dal bere acqua mentre mangi [...] perché mettere acqua sul cibo raffredda lo stomaco, spegne il calore della digestione e si mescola al cibo, e se se ne beve molta produce un blocco, e non c'è niente di peggio per il corpo" [Similiter retine animum tuum a potacione aque super cibum [...] quia potacio aque super cibum refrigerat stomachum et extinguit *ignem voluntatis et confundit cibum et generat impedimentum si multum bibatur, quo nichil corpori deterius est]. Avverto che, contaminando, ho tradotto lo strano ignem voluntatis che s'incontra nel testo della traduzione di Giovanni di Siviglia pubblicato dalla Spetia (in altri testimoni è l'ignem voluptatis dell'ed. Suchier, come ricavo dall'apparato della medesima Spetia) con il digestionis calorem che gli corrisponde nella traduzione integrale del Secretum secretorum (opera araba del X secolo, del quale l'Epistola è un estratto) di Filippo di Tripoli, ed. Möller. 
Se stessi male, non crederei certo a chi mi dicesse che sono sano, né, essendo sano, crederei a chi dicesse che sono malato. Il mio fuocherello interno è ancora abbastanza forte da cuocere ciò che gli è affidato, e anche qualcosa di più, se occorre. $^{78}$

E, poco oltre, torna a toccare il merito della questione. L'età lo ha cambiato, è vero, ma nella quantità, non nella qualità, sì che quella teoria degli elementi applicata ai cibi e agli umori che prevede una continua pratica di compensazione continua a restare sostanzialmente valida:

Inoltre la mia natura è cambiata in quantità, non in qualità. Fui infatti caldissimo: ora caldissimo non sono più, ma sono tuttavia caldo. L'acqua è un elemento freddo, e la frutta freddissimo: ma i mali si curano con i loro contrari, secondo quella che sento essere la regola dei medici, onde, come un tempo era giusto usarne con qualche abbondanza, ora è necessario ridurne la quantità. Ed io ho fatto appunto l'una e l'altra cosa: vedi tu cosa ricavarne. ${ }^{79}$

Ma non è solo questo il punto, né occorre qui inseguire Petrarca nelle sue belle difese dell'acqua, della frutta e del digiuno. Notevole è invece che egli veda quelle sue abitudini non come pratiche isolate che possano essere messe in discussione una per una, ma come altrettanti capisaldi di un modo di essere che va preservato nella sua integrità, perché ne devono essere preservati i valori e i significati, anche simbolici. Ogni volta che egli riafferma, per esempio, il suo gusto per l'acqua fresca che gli avrebbe sempre garantito un eccellente stato di salute, intende anche riaffermare un ideale di vita che ha il suo cardine tanto fisico quanto morale nella fedeltà e nell'armonia con la natura, quasi che l'attingere alla pura acqua delle fonti equivalga a succhiare la vita stessa dalla madre terra. E riafferma pure, in trasparenza, il suo giudizio sulla curia avignonese e sui suoi ricchi e corrotti prelati che più dell'acqua amano il vino di Borgogna, per amore del quale s'oppongono al ritorno della sede papale a Roma. Ogni volta che esalta il profumo e il sapore della frutta che tanto ama e che sa descrivere con tanta precisione, ogni volta che racconta della sua giovanile avidità per il gusto acido delle mele non perfettamente mature, egli esalta insieme la spontanea fecondità della terra e ne celebra con stupita meraviglia i doni. E rinvia, tacitamente o meno, all'atroce mensa dell'occupatus, al suo oltraggioso spreco, alla sua barbarie, e a tutta l'ingiustizia di cui è imbandita... E si direbbe ancora che il tempo dell'acedia, in questo Petrarca vecchio e saggio, sia lontano, o almeno corretto da una dose final-

78. Opera omnia, p. 1007: «Ego autem sicut eger nulli sanum me dicenti, sic sanus nulli egrum affirmanti credam. Igniculus quidem meus potens est sibi commissa percoquere plusque aliquid si necesse sit».

79. Opera omnia, p. 1008: «Ad hec, mutata est natura in quanto, non in quali. Calidissimus enim fui: non iam calidissimus sed adhuc calidus sum. Aqua est frigidum elementum, et poma sunt gelida: contraria vero contrariis curari medicorum, ut audio, lex est, quamobrem sicut his fuit aliquanto profusius, sic nunc parcius est utendum. Utrumque autem factum est: quid seguatur nosti». Per la teoria degli elementi, vedi sopra, nota 42. 
mente sincera di semplice gusto per la vita. Donde anche l'importanza che assume la conoscenza della propria natura - la natura del proprio corpoe delle sue specifiche esigenze. Se c'è una posizione che Petrarca continuamente ribadisce, in queste lettere, e sulla quale non è disposto a recedere, è proprio questa: qualche prescrizione medica la si può anche accettare, ma solo dopo che egli l'abbia giudicata e approvata sulla base di quella esperienza di sé alla quale spetta l'ultima parola, e che ad ogni medico sia pur eccellente nel suo mestiere (ma, chiede Petrarca, ne esistono?) resterà sempre inaccessibile. Che è un modo per dire, in buona sostanza, che la libertà del soggetto è intoccabile, per quanto malato o semplicemente indebolito egli sia, e che altrettanto intoccabili sono, ormai, le scelte - tutte le scelte- che l'hanno fatto libero. Ed è anche un modo per significare che in ogni caso, per definizione, la responsabilità della cura di sé è strettamente personale, né ad essa è possibile abdicare, ed anzi merita il rischio che per affermarla si beva un poco più d'acqua o si mangi qualche frutto o erbaggio in più di quanto il medico vorrebbe. Petrarca non difende dunque né il suo capriccio, né il mito ingenuo e scoraggiante di un "ritorno alla natura»: difende piuttosto, in uno dei suoi tanti avamposti, un'identità lungamente costruita e l'insieme dei suoi valori. Difende le sue scelte e la sua storia: e la scelta del cibo diventa, in questo contesto, un tratto distintivo in più, e non dei secondari. Proprio per questo, infine - per il «valore aggiunto» che il cibo assume quando in esso si condensa l'immagine d'una scelta di vita - il fatto che la sua dieta fosse un po' più varia e magari un po' più abbondante, e che alquanto più larga fosse la sua accettazione dei consigli dei medici (cose che, si badi, egli stesso ci fa capire) non toglie nulla al suo impegno e alla forza del modello proposto. Che è il modello di un uomo che si vuole capace di coniugare l'etica con la bellezza, la ricchezza con lo stile, e il cibo con la naturale eleganza del vivere. 